


\title{
Expedient Synthesis of N1-Substituted Triazole Peptidomimetics
}

\author{
Steven B. Coffey, Gary Aspnes, Allyn T. Londregan \\ Pfizer Inc. Worldwide Medicinal Chemistry, Eastern Point Rd., Groton, Connecticut, 06340 USA
}

\section{Supporting Information}

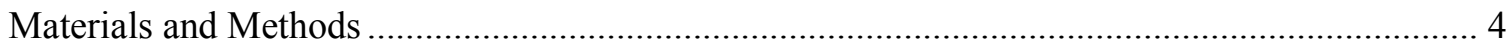

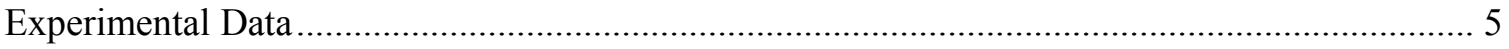

General procedure for the preparation of alkyne substrates (Scheme 2): benzyl (2,2dimethylbut-3-ynoyl)-L-leucyl-L-valyl-L-phenylalaninate (8a) ............................................. 5

Methyl (2,2-dimethylbut-3-ynoyl)-L-leucyl-L-valyl-L-tyrosinate (8b) ............................... 5

Benzyl(2,2-dimethylbut-3-ynoyl)-L-phenylalanyl-L-alanyl-L-prolinate $(8 \mathrm{c}) \ldots \ldots \ldots \ldots \ldots \ldots \ldots \ldots$

Benzyl (2,2-dimethylbut-3-ynoyl)-L-alanyl-L-alanyl-L-prolinate (8d) ................................. 6

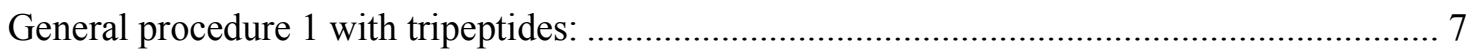

Benzyl(2-methyl-2-(1-(pyridin-3-yl)-1H-1,2,3-triazol-4-yl)propanoyl)-L-leucyl-L-valyl-L-

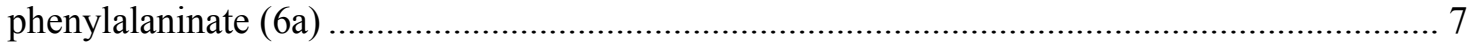

Benzyl(2-(1-(6-methoxypyridin-3-yl)-1H-1,2,3-triazol-4-yl)-2-methylpropanoyl)-L-leucyl-

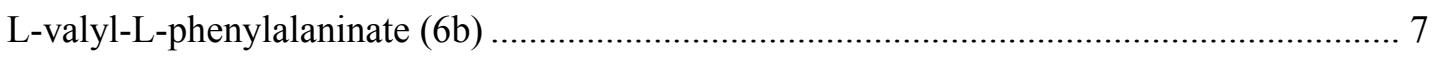

Benzyl(2-methyl-2-(1-(quinolin-6-yl)-1H-1,2,3-triazol-4-yl)propanoyl)-L-leucyl-L-valyl-Lphenylalaninate $(6 \mathrm{e})$ 8

Benzyl(2-methyl-2-(1-(1-((2-(trimethylsilyl)ethoxy)methyl)-1H-benzo[d]imidazol-5-yl)-1H1,2,3-triazol-4-yl)propanoyl)-L-leucyl-L-valyl-L-phenylalaninate (6i).....

Benzyl(2-(1-(1H-benzo[d]imidazol-5-yl)-1H-1,2,3-triazol-4-yl)-2-methylpropanoyl)-L-

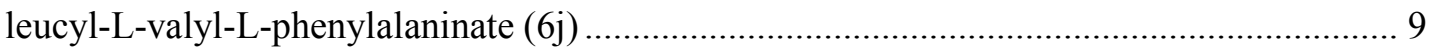

Benzyl(2-(1-benzyl-1H-1,2,3-triazol-4-yl)-2-methylpropanoyl)-L-leucyl-L-valyl-Lphenylalaninate $(6 \mathrm{p})$

Benzyl(2-methyl-2-(1-phenethyl-1H-1,2,3-triazol-4-yl)propanoyl)-L-leucyl-L-valyl-Lphenylalaninate $(6 \mathrm{t})$

Benzyl(2-(1-(2-(benzofuran-3-yl)ethyl)-1H-1,2,3-triazol-4-yl)-2-methylpropanoyl)-L-leucylL-valyl-L-phenylalaninate $(6 x)$

Benzyl(2-(1-hexyl-1H-1,2,3-triazol-4-yl)-2-methylpropanoyl)-L-leucyl-L-valyl-Lphenylalaninate $(6 y)$ 
tert-butyl3-((4-((4S,7R,10S)-4-benzyl-10-isobutyl-7-isopropyl-13-methyl-3,6,9,12-tetraoxo1-phenyl-2-oxa-5,8,11-triazatetradecan-13-yl)-1H-1,2,3-triazol-1-yl)methyl)pyrrolidine-1-

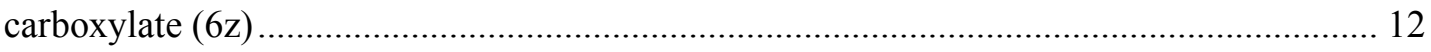

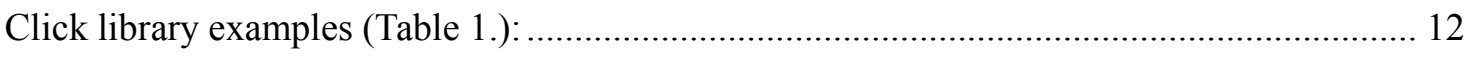

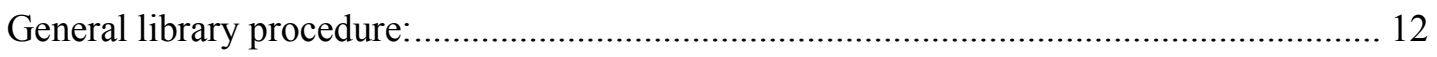

Methyl(2-(1-(6-methoxypyridin-3-yl)-1H-1,2,3-triazol-4-yl)-2-methylpropanoyl)-L-leucyl-

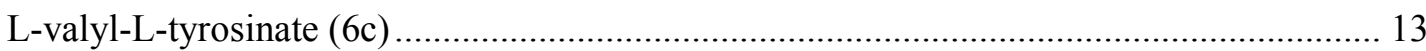

Benzyl(2-(1-(6-methoxypyridin-3-yl)-1H-1,2,3-triazol-4-yl)-2-methylpropanoyl)-L-

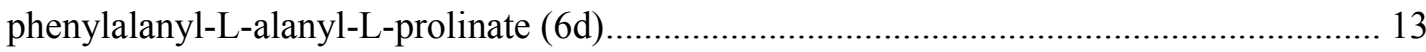

Benzyl(2-methyl-2-(1-(quinolin-6-yl)-1H-1,2,3-triazol-4-yl)propanoyl)-L-leucyl-L-valyl-L-

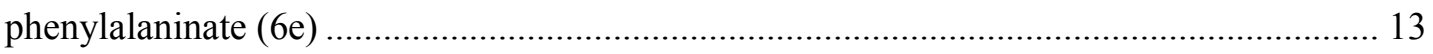

Methyl(2-methyl-2-(1-(quinolin-6-yl)-1H-1,2,3-triazol-4-yl)propanoyl)-L-leucyl-L-valyl-L-

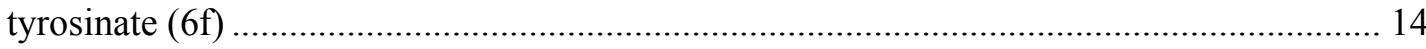

Benzyl(2-methyl-2-(1-(quinolin-6-yl)-1H-1,2,3-triazol-4-yl)propanoyl)-L-phenylalanyl-L-

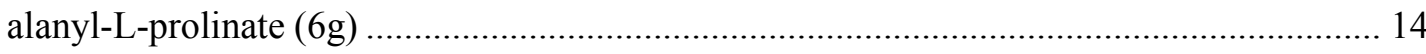

Benzyl (2-methyl-2-(1-(quinolin-6-yl)-1H-1,2,3-triazol-4-yl)propanoyl)-D-alanyl-L-alanylL-prolinate (6h) 15

Methyl(2-(1-(1H-benzo[d]imidazol-5-yl)-1H-1,2,3-triazol-4-yl)-2-methylpropanoyl)-L-

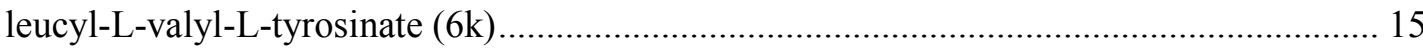

Benzyl(2-(1-(1H-benzo[d]imidazol-5-yl)-1H-1,2,3-triazol-4-yl)-2-methylpropanoyl)-L-

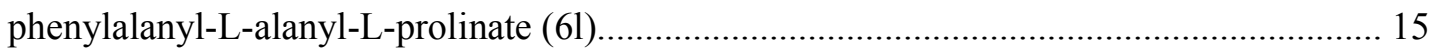

Benzyl(2-methyl-2-(1-(2-methylpyridin-4-yl)-1H-1,2,3-triazol-4-yl)propanoyl)-L-leucyl-D-

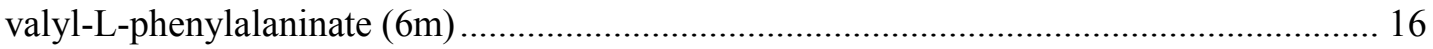

Methyl(2-methyl-2-(1-(2-methylpyridin-4-yl)-1H-1,2,3-triazol-4-yl)propanoyl)-L-leucyl-L-

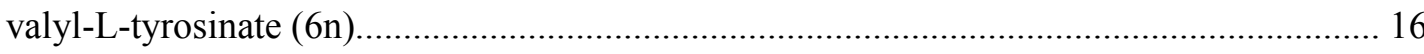

Benzyl(2-methyl-2-(1-(2-methylpyridin-4-yl)-1H-1,2,3-triazol-4-yl)propanoyl)-Lphenylalanyl-L-alanyl-L-prolinate (6o)

Benzyl(2-(1-benzyl-1H-1,2,3-triazol-4-yl)-2-methylpropanoyl)-L-leucyl-L-valyl-Lphenylalaninate $(6 \mathrm{p})$

Methyl(2-(1-benzyl-1H-1,2,3-triazol-4-yl)-2-methylpropanoyl)-L-leucyl-L-valyl-Ltyrosinate $(6 q)$.

Benzyl(2-(1-benzyl-1H-1,2,3-triazol-4-yl)-2-methylpropanoyl)-L-phenylalanyl-L-alanyl-Lprolinate $(6 r)$.

Benzyl(2-(1-benzyl-1H-1,2,3-triazol-4-yl)-2-methylpropyl)-L-alanyl-L-alanyl-L-prolinate $(6 s)$

Methyl(2-methyl-2-(1-phenethyl-1H-1,2,3-triazol-4-yl)propanoyl)-L-leucyl-L-valyl-Ltyrosinate $(6 u)$. 
Benzyl(2-methyl-2-(1-phenethyl-1H-1,2,3-triazol-4-yl)propanoyl)-L-phenylalanyl-L-alanyl-

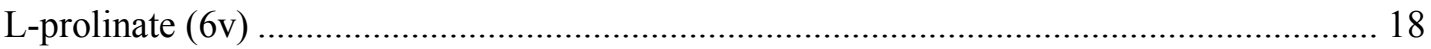

Benzyl(2-methyl-2-(1-phenethyl-1H-1,2,3-triazol-4-yl)propanoyl)-L-alanyl-L-alanyl-L-

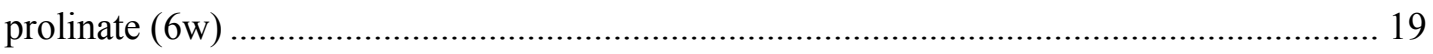

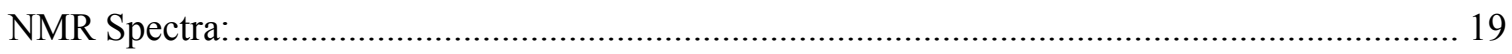




\section{Materials and Methods}

All chemicals, reagents, and solvents were purchased from commercial sources when available and used without further purification. The ligand (1S,2S)-N1,N2-dimethylcyclohexane-1,2diamine was purchased from Aldrich. Air and moisture-insensitive reactions were carried out under an ambient atmosphere, magnetically stirred, and monitored by thin layer chromatography (TLC) using pre-coated Whatman $250 \mu \mathrm{m}$ silica gel plates and visualized by fluorescence quenching under UV light. Reactions were carried out as described in Experimental Data. Silica gel chromatography was performed using an ISCO or Biotage system using ISCO or Biotage prepackaged columns. Reverse phase HPLC purification was performed using an Atlantis dC18 5 um $4.6 \times 50 \mathrm{~mm}$ column with an acetonitrile/water $0.05 \%$ TFA mobile phase. Concentration under reduced pressure (in vacuo) was performed by rotary evaporation at $25-30^{\circ} \mathrm{C}$ with appropriate pressure or Genevac centrifugal evaporators. Purified compounds were further dried under high vacuum to remove residual solvent. Yields refer to purified compounds. NMR spectra were recorded with a Agilent Unity I spectrometer equipped with an AutoX ID 600-5 probe at 600 $\mathrm{MHz}$ and $151 \mathrm{MHz}$ for ${ }^{1} \mathrm{H},{ }^{13} \mathrm{C}$ acquisitions, respectively. Chemical shifts were referenced to the residual ${ }^{1} \mathrm{H}$ solvent signals $\left(\mathrm{CDCl}_{3}, \delta 7.27\right)$ and solvent ${ }^{13} \mathrm{C}$ signals $\left(\mathrm{CDCl}_{3}, \delta 77.0\right)$. Signals are listed as follows: chemical shift in ppm (multiplicity identified as $\mathrm{s}=$ singlet, $\mathrm{br}=$ broad, $\mathrm{d}=$ doublet, $\mathrm{t}=$ triplet, $\mathrm{q}=$ quartet, $\mathrm{m}=$ multiplet; coupling constants in $\mathrm{Hz}$; integration). High resolution mass spectrometry (HRMS) was performed via atmospheric pressure chemical ionization (APCI) or electron scatter ionization (ESI) sources. 


\section{Experimental Data}

General procedure for the preparation of alkyne substrates (Scheme 2): benzyl (2,2-dimethylbut-3-ynoyl)-L-leucyl-L-valyl-L-phenylalaninate (8a)<smiles>C#CC(C)(C)C(=O)OCC#CC(C)(C)C(=O)N[C@@H](CC(C)C)C(=O)N[C@H](C(=O)N[C@H](Cc1ccccc1)C(=O)OCc1ccccc1)C(C)C</smiles>

In an $8 \mathrm{ml}$ vial was dissolved benzyl leucylvalylphenylalaninate (111 $\mathrm{mg}, 0.22 \mathrm{mmol}), 2,2-$ dimethylbut-3-ynoic acid (25 mg, $0.22 \mathrm{mmol}$ ), DIPEA (116 ul, $0.66 \mathrm{mmol}$ ), HBTU ( $85 \mathrm{mg}, 0.223$ $\mathrm{mmol}$ ) into $4 \mathrm{ml}$ DMF. The reaction stirred at ambient temperature for $16 \mathrm{hr}$. At which time the reaction was concentrated, dissolved into ethyl acetate and washed with sat. NaHCO3, brine, dried over Na2SO4, filtered and concentrated to a colorless oil $238 \mathrm{mg}$. TLC in 7:3 ethyl acetate/heptane shows single spot $\mathrm{Rf}=0.6$. Purified using a $5 \mathrm{~g}$ silica pre-column and a $12 \mathrm{~g}$ RediSep Gold silica analytical column with a $0-50 \%$ ethyl acetate/heptane gradient over 28 CV. Concentrated to a white foam and dried on vac pump to $113 \mathrm{mg}(90 \%)$. ${ }^{1} \mathrm{H}$ NMR (400 MHz, CHLOROFORM-d) $\delta$ 7.34-7.40 (m, 3H), 7.28-7.32 (m, 2H), 7.21-7.25 (m, 2H), 7.02 (dd, J=2.93, $6.83 \mathrm{~Hz}, 3 \mathrm{H}), 6.60-6.65(\mathrm{~m}, 1 \mathrm{H}), 6.20-6.24(\mathrm{~m}, 1 \mathrm{H}), 5.14$ (d, J=14.05 Hz, 2H), 4.88-4.96 (m, 1H), 4.32-4.41 (m, 1H), 4.17-4.23 (m, 1H), 3.12 (d, $J=5.85 \mathrm{~Hz}, 2 \mathrm{H}), 2.50(\mathrm{~s}, 1 \mathrm{H}), 1.57-1.77$ (m, 3H), $1.47(\mathrm{~d}, J=9.37 \mathrm{~Hz}, 5 \mathrm{H}), 0.82-0.98(\mathrm{~m}, 12 \mathrm{H})$. LCMS (m/z): calculated for $\mathrm{C}_{33} \mathrm{H}_{44} \mathrm{~N}_{3} \mathrm{O}_{5}[\mathrm{M}+\mathrm{H}]^{+}$ 562 ; found 562.3 .

\section{Methyl (2,2-dimethylbut-3-ynoyl)-L-leucyl-L-valyl-L-tyrosinate (8b)}<smiles>C#CC(C)(C)C(=O)N[C@@H](CC(C)C)C(=O)N[C@H](C(=O)N[C@@H](Cc1ccc(O)cc1)C(=O)OC)C(C)C</smiles>

Following the general procedure, the title compound was prepared as a colorless solid $(64 \%)$ starting from methyl L-leucyl-L-valyl-L-tyrosinate. ${ }^{1} \mathrm{H}$ NMR (400 MHz, CHLOROFORM-d) $\delta$ 6.92-7.08 (m, 3H), 6.71-6.81 (m, 2H), 6.59-6.69 (m, 1H), $6.25(\mathrm{~s}, 1 \mathrm{H}), 5.67(\mathrm{~s}, 1 \mathrm{H}), 4.83(\mathrm{~d}$, $J=8.20 \mathrm{~Hz}, 1 \mathrm{H}), 4.34-4.45(\mathrm{~m}, 1 \mathrm{H}), 4.22$ (dd, $J=6.24,8.59 \mathrm{~Hz}, 1 \mathrm{H}), 3.74$ (s, 3H), 3.04 (dd, $J=5.85$, $16.00 \mathrm{~Hz}, 2 \mathrm{H}), 2.51$ (s, 1H), 2.07-2.18 (m, 1H), 1.76 (s, 3H), 1.48 (d, J=8.98 Hz, 6H), 0.83- 
$1.00(\mathrm{~m}, 12 \mathrm{H})$. LCMS (m/z): calculated for $\mathrm{C}_{27} \mathrm{H}_{40} \mathrm{~N}_{3} \mathrm{O}_{6}[\mathrm{M}+\mathrm{H}]^{+}$502; found 502.3.

\section{Benzyl(2,2-dimethylbut-3-ynoyl)-L-phenylalanyl-L-alanyl-L-prolinate (8c)}

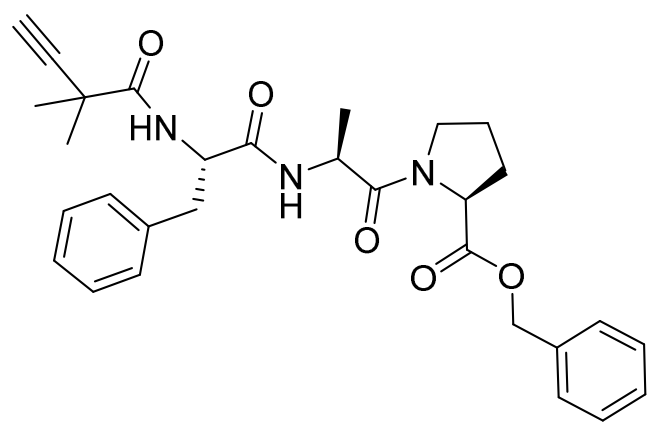

Following the general procedure, the title compound was prepared as a colorless solid (52\%) starting from benzyl phenylalanylalanylprolinate. ${ }^{1} \mathrm{H}$ NMR (400 MHz, CHLOROFORM-d) $\delta$ 7.28-7.40 (m, 6H), 7.13-7.26 (m, 4H), 6.66-6.72 (m, 1H), 5.07-5.23 (m, 2H), $4.64(\mathrm{~s}, 3 \mathrm{H}), 3.53-$ 3.69 (m, 2H), 3.12 (dd, $J=4.49,6.44 \mathrm{~Hz}, 2 \mathrm{H}), 2.43$ (s, 1H), 2.01 (d, $J=3.51 \mathrm{~Hz}, 3 \mathrm{H}), 1.43-1.46$ (m, $3 \mathrm{H}), 1.28-1.38(\mathrm{~m}, 7 \mathrm{H})$. ). LCMS (m/z): calculated for $\mathrm{C}_{30} \mathrm{H}_{36} \mathrm{~N}_{3} \mathrm{O}_{5}[\mathrm{M}+\mathrm{H}]^{+}$518; found 518.2.

\section{Benzyl (2,2-dimethylbut-3-ynoyl)-L-alanyl-L-alanyl-L-prolinate (8d)}<smiles>C#CC(C)(C)C(=O)N[C@@H](C)C(=O)N[C@@H](C)C(=O)N1CCCC1C(=O)OCc1ccccc1</smiles>

Following the general procedure, the title compound was prepared as a colorless solid $(64 \%)$ starting from benzyl alanylalanylprolinate . ${ }^{1} \mathrm{H}$ NMR (400 MHz, CHLOROFORM-d) $\delta$ 7.28-7.41 (m, 6H), 5.07-5.26 (m, 2H), $4.70(\mathrm{t}, J=7.02 \mathrm{~Hz}, 1 \mathrm{H}), 4.60$ (dd, $J=4.29,8.98 \mathrm{~Hz}, 1 \mathrm{H}), 4.40$ (t, $J=7.02 \mathrm{~Hz}, 1 \mathrm{H}), 3.55-3.74(\mathrm{~m}, 2 \mathrm{H}), 2.81(\mathrm{~s}, 1 \mathrm{H}), 2.51(\mathrm{~s}, 1 \mathrm{H}), 2.17-2.30(\mathrm{~m}, 1 \mathrm{H}), 1.96-2.05(\mathrm{~m}$, 2H), 1.47 (d, $J=3.12 \mathrm{~Hz}, 6 \mathrm{H}), 1.40$ (d, $J=7.02 \mathrm{~Hz}, 3 \mathrm{H}), 1.34$ (d, $J=7.02 \mathrm{~Hz}, 3 \mathrm{H})$. LCMS (m/z): calculated for $\mathrm{C}_{24} \mathrm{H}_{31} \mathrm{~N}_{3} \mathrm{O}_{5}[\mathrm{M}+\mathrm{H}]^{+} 442$; found 442 . 


\section{General procedure 1 with tripeptides:}

\section{Benzyl(2-methyl-2-(1-(pyridin-3-yl)-1H-1,2,3-triazol-4-yl)propanoyl)-L- leucyl-L-valyl-L-phenylalaninate (6a)}

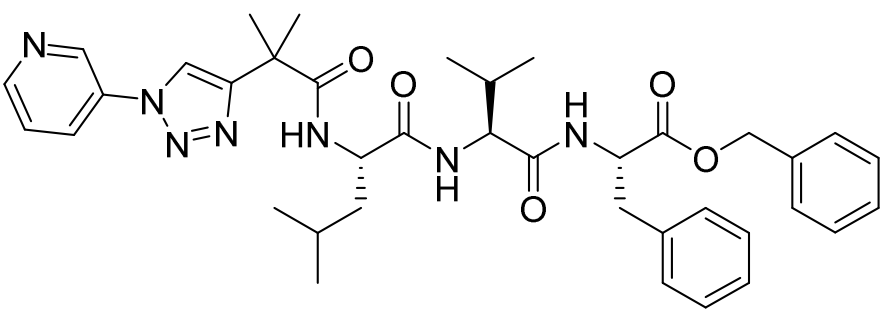

To the aryl bromide $(0.061 \mathrm{mmol})$, was added $320 \mathrm{ul}$ of a solution of: sodium azide $(4.2 \mathrm{mg}$, $0.063 \mathrm{mmol})$, the alkyne $(34 \mathrm{mg}, 0.061 \mathrm{mmol})$, sodium ascorbate $(1.2 \mathrm{mg}, 0.006 \mathrm{mmol})$, CuI (1.1 $\mathrm{mg}, 0.007 \mathrm{mmol})$, N1,N2-dimethylcyclohexane-1,2-diamine $(1.3 \mathrm{mg}, 0.009 \mathrm{mmol})$ and 265 ul DMSO, 55 ul water (5:1). Degassed and filled with nitrogen 3x's, the reaction stirred at 70C. After heating for 4 hours, the reaction was cooled to RT. Added $1 \mathrm{ml}$ ice cooled water, extracted with ethyl acetate and concentrated to an oil. The crude oil was purified using a $5 \mathrm{~g}$ silica pre-column and a $12 \mathrm{~g}$ silica RediSep Gold analytical column with a 0-100\% ethyl acetate in heptane mobile phase. Combined and concentrated like fractions. The title compound was prepared as a colorless oil $29 \mathrm{mg}(70 \%)$ starting from 3-bromopyridine. ${ }^{1} \mathrm{H}$ NMR $(600 \mathrm{MHz}$, CHLOROFORM-d) $\delta 9.04$ (br. s., 1H), 8.71 (br. s., 1H), 8.14 (d, $J=8.22 \mathrm{~Hz}, 1 \mathrm{H}), 8.01$ (s, 1H), 7.50 (dd, $J=4.99,7.92 \mathrm{~Hz}, 1 \mathrm{H}$ ), 7.31-7.37 (m, 3H), 7.26 (br. s., 1H), 7.16-7.23 (m, 3H), 6.96-7.04 $(\mathrm{m}, 3 \mathrm{H}), 6.73(\mathrm{~d}, J=8.80 \mathrm{~Hz}, 1 \mathrm{H}), 6.48(\mathrm{~d}, J=7.63 \mathrm{~Hz}, 1 \mathrm{H}), 5.06-5.16(\mathrm{~m}, 2 \mathrm{H}), 4.83-4.88(\mathrm{~m}, 1 \mathrm{H})$, 4.37-4.44 (m, 1H), 4.21 (dd, $J=6.16,8.51 \mathrm{~Hz}, 1 \mathrm{H}), 3.09$ (d, $J=5.87 \mathrm{~Hz}, 2 \mathrm{H}), 2.04$ (qd, $J=6.50$, $13.35 \mathrm{~Hz}, 1 \mathrm{H}), 1.66-1.75(\mathrm{~m}, 7 \mathrm{H}), 1.53-1.66(\mathrm{~m}, 2 \mathrm{H}), 0.85-0.95$ (m, 6H), 0.78 (dd, $J=7.04,19.37$ $\mathrm{Hz}, 6 \mathrm{H}) . \quad{ }^{13} \mathrm{C}$ NMR (151 MHz, CHLOROFORM-d) $\delta 174.9,171.8,171.0,170.4,153.4,149.9$, $141.5,135.5,135.0,129.2,128.5,128.2,127.1,124.2$, 119.2, 67.2, 58.1, 53.1, 52.5, 41.8, 40.1, 37.8, 30.7, 26.5, 26.1, 24.9, 23.0, 21.7, 19.0, 17.6. HRMS (m/z): calculated for $\mathrm{C}_{38} \mathrm{H}_{47} \mathrm{~N}_{7} \mathrm{NaO}_{5}$ $[\mathrm{M}+\mathrm{Na}]^{+} 704.3531$; found 704.3538 .

\section{Benzyl(2-(1-(6-methoxypyridin-3-yl)-1H-1,2,3-triazol-4-yl)-2- methylpropanoyl)-L-leucyl-L-valyl-L-phenylalaninate (6b)}

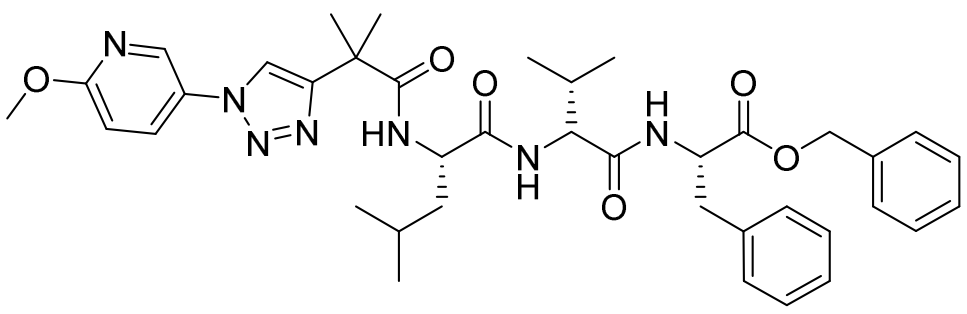

Following GP1, the title compound was prepared as a colorless solid (80\%) starting from 5- 
bromo-2-methoxypyridine. ${ }^{1} \mathrm{H}$ NMR (400 MHz, CHLOROFORM-d) $\delta 8.49$ (d, $\left.J=2.34 \mathrm{~Hz}, 1 \mathrm{H}\right)$, 7.94 (dd, $J=2.93,8.78 \mathrm{~Hz}, 1 \mathrm{H}), 7.83(\mathrm{~s}, 1 \mathrm{H}), 7.32-7.39$ (m, 3H), 7.26 (dd, $J=1.37,4.88 \mathrm{~Hz}, 1 \mathrm{H})$, 7.18-7.24 (m, 3H), 6.98-7.08 (m, 3H), 6.86-6.93 (m, 1H), $6.72(\mathrm{~d}, J=8.59 \mathrm{~Hz}, 1 \mathrm{H}), 6.43$ (d, $J=8.20 \mathrm{~Hz}, 1 \mathrm{H}), 5.11(\mathrm{q}, J=12.10 \mathrm{~Hz}, 2 \mathrm{H}), 4.82-4.90(\mathrm{~m}, 1 \mathrm{H}), 4.35-4.43(\mathrm{~m}, 1 \mathrm{H}), 4.19$ (dd, $J=5.85,8.59 \mathrm{~Hz}, 1 \mathrm{H}), 4.00$ (s, 3H), 3.09 (d, $J=5.85 \mathrm{~Hz}, 2 \mathrm{H}), 2.06$ (d, $J=6.24 \mathrm{~Hz}, 1 \mathrm{H}), 1.87$ (s, 1H), 1.66-1.77 (m, 7H), 1.52-1.66 (m, 2H), 0.90 (dd, $J=6.44,13.85 \mathrm{~Hz}, 6 \mathrm{H}), 0.78$ (dd, $J=6.83,14.63$ $\mathrm{Hz}, 6 \mathrm{H}) .{ }^{13} \mathrm{C}$ NMR (101 MHz, CHLOROFORM-d) $\delta 175.1,171.9,171.0,170.4,164.1,152.9$, $139.3,135.5,135.0,132.0,129.2$, 128.5, 127.1, 119.4, 111.7, 67.2, 58.1, 54.1, 53.1, 52.5, 41.8, $39.9,37.8,30.6,26.3,24.9,23.0,21.7,19.0,17.5$. LCMS (m/z): calculated for $\mathrm{C}_{39} \mathrm{H}_{50} \mathrm{~N}_{7} \mathrm{O}_{6}[\mathrm{M}+$ $\mathrm{H}]^{+} 712$; found 712.4 .

\section{Benzyl(2-methyl-2-(1-(quinolin-6-yl)-1H-1,2,3-triazol-4-yl)propanoyl)- L-leucyl-L-valyl-L-phenylalaninate (6e)}

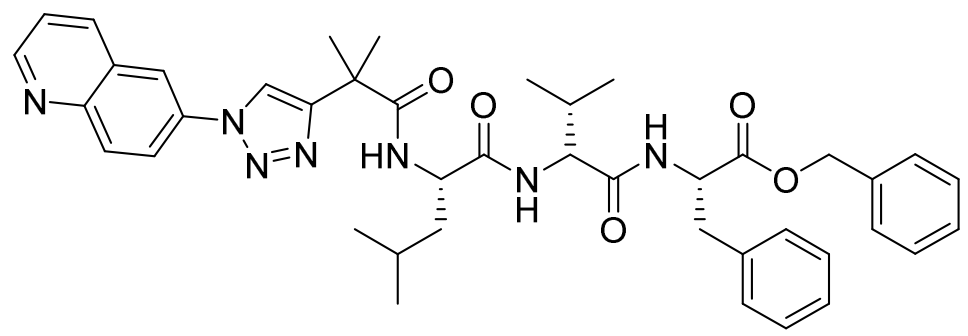

Following GP1, the title compound was prepared as a colorless solid (62\%) starting from 6bromoquinoline. ${ }^{1} \mathrm{H}$ NMR (400 MHz, CHLOROFORM-d) $\delta 9.00$ (dd, $J=1.95,4.29 \mathrm{~Hz}, 1 \mathrm{H}$ ), 8.24-8.33 (m, 2H), 8.21 (d, $J=2.34 \mathrm{~Hz}, 1 \mathrm{H}), 8.13$ (dd, $J=2.34,8.98 \mathrm{~Hz}, 1 \mathrm{H}), 8.07$ (s, 1H), 7.51 (dd, $J=4.29,8.20 \mathrm{~Hz}, 1 \mathrm{H}), 7.31-7.38(\mathrm{~m}, 3 \mathrm{H}), 7.25(\mathrm{dd}, J=2.93,6.83 \mathrm{~Hz}, 2 \mathrm{H}), 7.16-7.23(\mathrm{~m}, 3 \mathrm{H})$, 6.98-7.09 (m, 3H), 6.73 (d, $J=8.59 \mathrm{~Hz}, 1 \mathrm{H}), 6.42(\mathrm{~d}, J=7.80 \mathrm{~Hz}, 1 \mathrm{H}), 5.10$ (q, J=12.10 Hz, 2H), 4.87 (td, $J=5.95,8.00 \mathrm{~Hz}, 1 \mathrm{H}), 4.40$ (ddd, $J=5.07,7.41,9.37 \mathrm{~Hz}, 1 \mathrm{H}), 4.20$ (dd, $J=5.85,8.59 \mathrm{~Hz}$, $1 \mathrm{H}), 3.09$ (d, $J=5.85 \mathrm{~Hz}, 2 \mathrm{H}), 2.00-2.12(\mathrm{~m}, 1 \mathrm{H}), 1.86(\mathrm{~s}, 1 \mathrm{H}), 1.68-1.79(\mathrm{~m}, 7 \mathrm{H}), 1.54-1.68$ (m, 2H), 0.85-0.97 (m, 6H), 0.79 (dd, $J=6.83,13.46 \mathrm{~Hz}, 6 \mathrm{H}) .{ }^{13} \mathrm{C}$ NMR (101 MHz, CHLOROFORMd) $\delta 175.1,171.9,171.0,170.4,153.1,151.5,147.7,136.3,135.5,135.0,134.6,131.7,129.2$, $128.5,128.2,127.1,122.5,122.2,119.3,118.3,67.2,58.2,53.1,52.5,41.8,39.9,37.8,30.6,26.3$, 24.9, 23.0, 21.7, 19.0, 17.6. LCMS (m/z): calculated for $\mathrm{C}_{42} \mathrm{H}_{50} \mathrm{~N}_{7} \mathrm{O}_{5}[\mathrm{M}+\mathrm{H}]^{+} 732$; found 732.4 


\section{Benzyl(2-methyl-2-(1-(1-((2-(trimethylsilyl)ethoxy)methyl)-1H- benzo[d]imidazol-5-yl)-1H-1,2,3-triazol-4-yl)propanoyl)-L-leucyl-L- valyl-L-phenylalaninate (6i)}

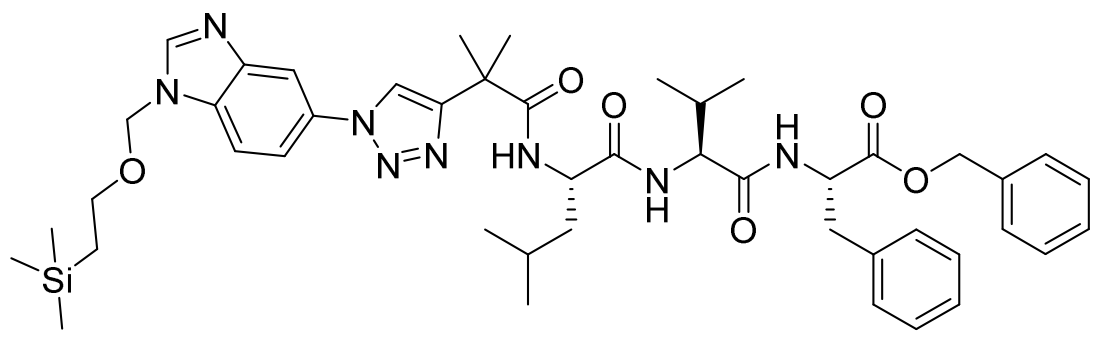

Following GP1, the title compound was prepared as a colorless oil (58\%) starting from 5-bromo1-((2-(trimethylsilyl)ethoxy)methyl)-1H-benzo[d]imidazole. $\quad{ }^{1} \mathrm{H} \quad$ NMR $\quad(400 \quad \mathrm{MHz}$, CHLOROFORM-d) $\delta 8.07-8.19(\mathrm{~m}, 1 \mathrm{H}), 7.98-8.03(\mathrm{~m}, 1 \mathrm{H}), 7.93(\mathrm{~d}, J=1.17 \mathrm{~Hz}, 1 \mathrm{H}), 7.75-7.80$ (m, 1H), 7.67-7.74 (m, 1H), 7.60-7.65 (m, 1H), 7.31-7.39 (m, 3H), 7.24-7.27 (m, 1H), 7.13-7.24 (m, 4H), 6.97-7.06 (m, 2H), 6.74-6.83 (m, 1H), 6.45-6.55 (m, 1H), $5.58(\mathrm{~d}, J=3.51 \mathrm{~Hz}, 2 \mathrm{H}), 5.11$ (dd, $J=1.76,10.73 \mathrm{~Hz}, 2 \mathrm{H}), 4.83-4.93(\mathrm{~m}, 1 \mathrm{H}), 4.33-4.45$ (m, 1H), 4.17-4.24 (m, 1H), 3.50-3.59 (m, 2H), $3.10(\mathrm{~d}, J=6.24 \mathrm{~Hz}, 2 \mathrm{H}), 2.06-2.16(\mathrm{~m}, 1 \mathrm{H}), 1.84-1.95(\mathrm{~m}, 1 \mathrm{H}), 1.68-1.78(\mathrm{~m}, 7 \mathrm{H}), 1.53-$ $1.67(\mathrm{~m}, 2 \mathrm{H}), 0.85-0.97(\mathrm{~m}, 8 \mathrm{H}), 0.74-0.84(\mathrm{~m}, 6 \mathrm{H}),-0.06--0.01(\mathrm{~m}, 9 \mathrm{H}) . \quad$ LCMS $(\mathrm{m} / \mathrm{z})$ : calculated for $\mathrm{C}_{46} \mathrm{H}_{62} \mathrm{~N}_{8} \mathrm{O}_{6} \mathrm{Si}[\mathrm{M}+\mathrm{H}]+851$; found 851.6 .

\section{Benzyl(2-(1-(1H-benzo[d]imidazol-5-yl)-1H-1,2,3-triazol-4-yl)-2- methylpropanoyl)-L-leucyl-L-valyl-L-phenylalaninate $(6 \mathbf{j})$}<smiles>CC(C)C[C@H](NC(=O)C(C)(C)c1cn(-c2ccc3[nH]cnc3c2)nn1)C(=O)N[C@@H](C(=O)N[C@@H](Cc1ccccc1)C(=O)OCc1ccccc1)C(C)C</smiles>

Following GP1, the title compound was prepared as a colorless oil (66\%) starting from 5-bromo benzimidazole. ${ }^{1} \mathrm{H}$ NMR (600 MHz, CHLOROFORM-d) $\delta$ 10.67-12.23 (m, 3H), 8.17 (br. s., 1H), 7.93 (br. s., 2H), 7.76 (br. s., 1H), 7.53 (br. s., 1H), 7.30 (br. s., 2H), 7.13-7.26 (m, 5H), 7.01 (d, $J=5.28 \mathrm{~Hz}, 2 \mathrm{H}), 6.83$ (br. s., $1 \mathrm{H}), 4.98-5.19$ (m, 2H), 4.85 (d, $J=6.46 \mathrm{~Hz}, 1 \mathrm{H}), 4.44$ (br. s., $1 \mathrm{H})$, 4.24 (br. s., 1H), 2.99-3.19 (m, 2H), 1.97-2.24 (m, 2H), 1.51-1.82 (m, 7H), 0.63-1.03 (m, 10H). ${ }^{13} \mathrm{C}$ NMR (151 MHz, CHLOROFORM-d) $\delta$ 175.6, 172.4, 171.1, 170.9, 152.7, 135.6, 134.9, 129.2 , 128.5, 127.1, 119.7, 100.0, 67.2, 58.5, 53.4, 52.8, 41.9, 40.1, 37.7, 30.6, 26.4, 25.0, 23.0, 21.6, 19.1, 17.7. HRMS (m/z): calculated for $\mathrm{C}_{40} \mathrm{H}_{48} \mathrm{~N}_{8} \mathrm{NaO}_{5}[\mathrm{M}+\mathrm{Na}]^{+}$743.364; found 743.3643. 


\section{Benzyl(2-(1-benzyl-1H-1,2,3-triazol-4-yl)-2-methylpropanoyl)-L-leucyl- L-valyl-L-phenylalaninate (6p)}

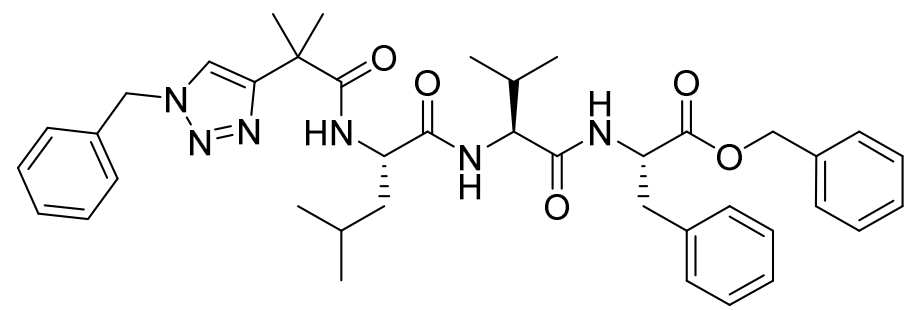

Following GP1, the title compound was prepared as a colorless solid (60\%) starting from benzylbromide. ${ }^{1} \mathrm{H}$ NMR (400 MHz, CHLOROFORM-d) $\delta$ 7.31-7.42 (m, 7H), 7.28-7.31 (m, 2H), 7.25-7.27 (m, 1H), 7.18-7.24 (m, 3H), 7.14 (d, $J=7.41 \mathrm{~Hz}, 1 \mathrm{H}), 6.99-7.08$ (m, 2H), 6.73 (d, $J=8.59 \mathrm{~Hz}, 1 \mathrm{H}), 6.44(\mathrm{~d}, J=8.20 \mathrm{~Hz}, 1 \mathrm{H}), 5.42-5.58(\mathrm{~m}, 2 \mathrm{H}), 5.12(\mathrm{q}, J=12.10 \mathrm{~Hz}, 2 \mathrm{H}), 4.88$ (td, $J=6.24,7.80 \mathrm{~Hz}, 1 \mathrm{H}), 4.32$ (ddd, $J=5.07,7.22,9.56 \mathrm{~Hz}, 1 \mathrm{H}), 3.10$ (d, $J=6.24 \mathrm{~Hz}, 2 \mathrm{H}), 1.82$ (br. s., $1 \mathrm{H}), 1.65-1.76(\mathrm{~m}, 1 \mathrm{H}), 1.48-1.64(\mathrm{~m}, 8 \mathrm{H}), 0.87-0.93(\mathrm{~m}, 4 \mathrm{H}), 0.84(\mathrm{~d}, J=6.63 \mathrm{~Hz}, 3 \mathrm{H}), 0.80$ (d, $J=6.63 \mathrm{~Hz}, 3 \mathrm{H}), 0.75(\mathrm{~d}, J=6.63 \mathrm{~Hz}, 3 \mathrm{H}) .{ }^{13} \mathrm{C}$ NMR (101 MHz, CHLOROFORM-d) $\delta 175.5$, $171.9,171.0,170.5,152.6,135.6,135.1,134.3,129.2,129.2,128.9,128.5,128.1,127.0,120.5$, $67.2,58.2,54.3,53.1,52.4,41.6,39.6,37.8,30.3,26.3,24.9,23.0,21.6,19.1,17.5$. LCMS $(\mathrm{m} / \mathrm{z})$ : calculated for $\mathrm{C}_{40} \mathrm{H}_{51} \mathrm{~N}_{6} \mathrm{O}_{5}[\mathrm{M}+\mathrm{H}]^{+}$695; found 695.5.

\section{Benzyl(2-methyl-2-(1-phenethyl-1H-1,2,3-triazol-4-yl)propanoyl)-L- leucyl-L-valyl-L-phenylalaninate (6t)}

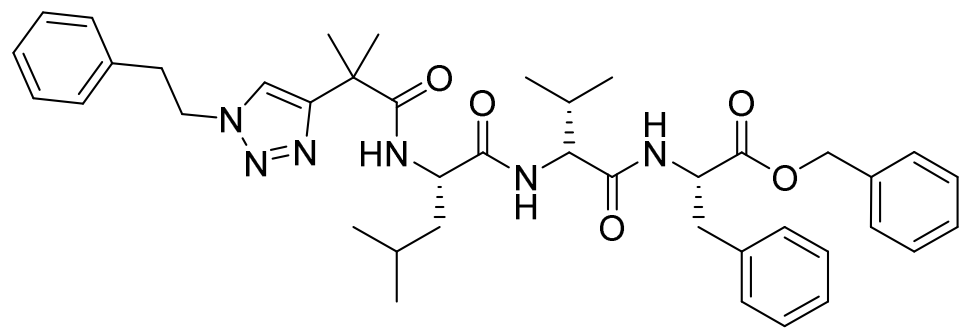

Following GP1, the title compound was prepared as a colorless oil (75\%) starting from (2bromoethyl)benzene. ${ }^{1} \mathrm{H}$ NMR (600 MHz, CHLOROFORM-d) $\delta$ 7.33-7.37 (m, 2H), 7.28-7.32 (m, 2H), 7.24-7.26 (m, 1H), 7.19-7.24 (m, 3H), $7.16(\mathrm{~d}, J=7.04 \mathrm{~Hz}, 1 \mathrm{H}), 7.12$ (s, 1H), 7.09 (d, $J=7.04$ $\mathrm{Hz}, 2 \mathrm{H}), 7.01-7.06(\mathrm{~m}, 2 \mathrm{H}), 6.74(\mathrm{~d}, J=8.80 \mathrm{~Hz}, 1 \mathrm{H}), 6.44(\mathrm{~d}, J=7.63 \mathrm{~Hz}, 1 \mathrm{H}), 5.04-5.19(\mathrm{~m}, 2 \mathrm{H})$, 4.81-4.92 (m, 1H), 4.47-4.65 (m, 2H), 4.30-4.38 (m, 1H), 4.18 (dd, $J=5.87,8.22 \mathrm{~Hz}, 1 \mathrm{H}), 3.20$ (t, $J=7.34 \mathrm{~Hz}, 2 \mathrm{H}), 3.10$ (d, $J=6.46 \mathrm{~Hz}, 2 \mathrm{H}), 2.11$ (qd, $J=6.80,13.06 \mathrm{~Hz}, 1 \mathrm{H}), 1.68-1.76(\mathrm{~m}, 1 \mathrm{H})$, 1.51-1.63 (m, 7H), 0.75-0.94 (m, 12H). ${ }^{13} \mathrm{C}$ NMR (151 MHz, CHLOROFORM-d) $\delta 175.5,171.9$, 171.0, 170.4, 151.8, 136.9, 135.6, 135.1, 129.2, 128.8, 128.5, 127.2, 127.0, 121.0, 67.1, 58.2, 53.1, 52.5, 51.8, 41.6, 39.7, 37.8, 36.7, 30.4, 26.4, 24.9, 22.3, 19.1, 17.5. HRMS (m/z): calculated for $\mathrm{C}_{41} \mathrm{H}_{53} \mathrm{~N}_{6} \mathrm{O}_{5}[\mathrm{M}+\mathrm{H}]^{+}$709.4072; found 709.4077. 


\section{Benzyl(2-(1-(2-(benzofuran-3-yl)ethyl)-1H-1,2,3-triazol-4-yl)-2- methylpropanoyl)-L-leucyl-L-valyl-L-phenylalaninate (6x)}

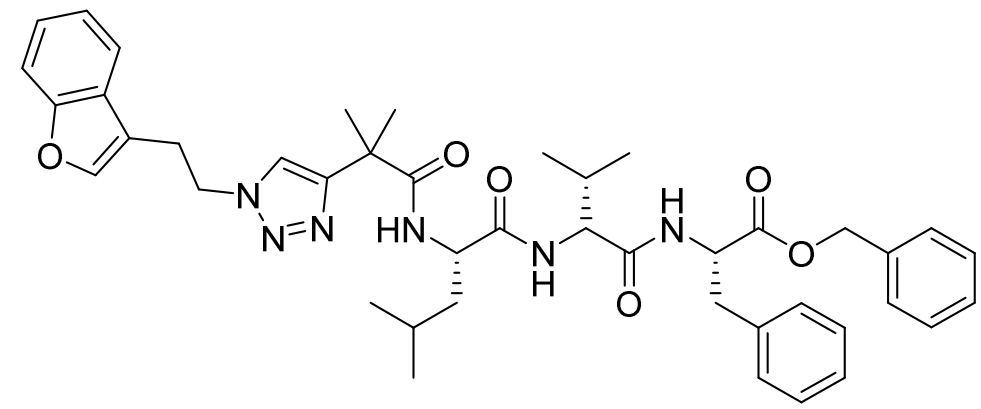

Following GP1, the title compound was prepared as a colorless oil (33 mg, 88\%) starting from 2(benzofuran-3-yl)ethyl 4-methylbenzenesulfonate and alkyne (1). Note: for purification, used $\mathrm{KMnO}_{4}$ stain for visualization of product. ${ }^{1} \mathrm{H}$ NMR (400 MHz, CHLOROFORM-d) $\delta 7.48$ (d, $J=8.20 \mathrm{~Hz}, 1 \mathrm{H}), 7.36-7.43(\mathrm{~m}, 2 \mathrm{H}), 7.29-7.36(\mathrm{~m}, 4 \mathrm{H}), 7.24-7.26(\mathrm{~m}, 2 \mathrm{H}), 7.17-7.24(\mathrm{~m}, 4 \mathrm{H})$, $7.11(\mathrm{~d}, J=7.41 \mathrm{~Hz}, 1 \mathrm{H}), 7.00-7.07(\mathrm{~m}, 2 \mathrm{H}), 6.73(\mathrm{~d}, J=8.20 \mathrm{~Hz}, 1 \mathrm{H}), 6.49$ (d, $J=7.80 \mathrm{~Hz}, 1 \mathrm{H})$, $5.10(\mathrm{q}, J=12.10 \mathrm{~Hz}, 2 \mathrm{H}), 4.87(\mathrm{td}, J=6.24,7.81 \mathrm{~Hz}, 1 \mathrm{H}), 4.55-4.73(\mathrm{~m}, 2 \mathrm{H}), 4.29-4.41(\mathrm{~m}, 1 \mathrm{H})$, 4.18 (dd, $J=6.24,8.59 \mathrm{~Hz}, 1 \mathrm{H}), 3.31$ (t, $J=7.02 \mathrm{~Hz}, 2 \mathrm{H}), 3.09$ (d, $J=6.24 \mathrm{~Hz}, 2 \mathrm{H}), 2.17-2.31$ (m, $1 \mathrm{H}), 2.08(\mathrm{td}, J=7.07,13.56 \mathrm{~Hz}, 1 \mathrm{H}), 1.66-1.77(\mathrm{~m}, 1 \mathrm{H}), 1.47-1.65(\mathrm{~m}, 8 \mathrm{H}), 0.84-0.95(\mathrm{~m}, 7 \mathrm{H})$, 0.80 (dd, $J=6.83,14.63 \mathrm{~Hz}, 6 \mathrm{H})$. HRMS (m/z): calculated for $\mathrm{C}_{43} \mathrm{H}_{53} \mathrm{~N}_{6} \mathrm{O}_{6}[\mathrm{M}+\mathrm{H}]^{+} 749.4021$; found 749.4042 .

\section{Benzyl(2-(1-hexyl-1H-1,2,3-triazol-4-yl)-2-methylpropanoyl)-L-leucyl-L- valyl-L-phenylalaninate $(6 y)$}

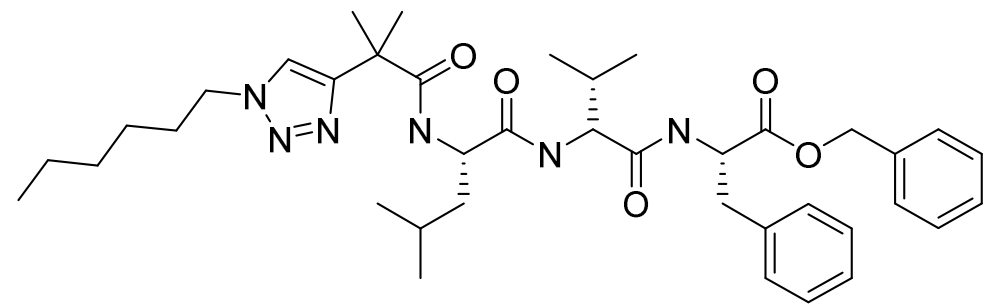

Following GP1, the title compound was prepared as a colorless oil (23 mg, 61\%) starting from hexyl 4-methylbenzenesulfonate and alkyne (1). Note: for purification, used $\mathrm{KMnO}_{4}$ stain for visualization of product. ${ }^{1} \mathrm{H}$ NMR (400 MHz, CHLOROFORM-d) $\delta$ 7.38-7.41 (m, 1H), 7.32-7.37 (m, 3H), 7.27-7.30 (m, 1H), 7.25-7.27 (m, 1H), 7.17-7.25 (m, 4H), 6.96-7.09 (m, 2H), 6.74 (d, 
$J=8.59 \mathrm{~Hz}, 1 \mathrm{H}), 6.45(\mathrm{~d}, J=8.20 \mathrm{~Hz}, 1 \mathrm{H}), 5.12(\mathrm{q}, J=12.10 \mathrm{~Hz}, 2 \mathrm{H}), 4.82-4.95(\mathrm{~m}, 1 \mathrm{H}), 4.27-4.40$ (m, 3H), 4.18 (dd, $J=5.85,8.59 \mathrm{~Hz}, 1 \mathrm{H}), 3.10$ (d, $J=6.24 \mathrm{~Hz}, 2 \mathrm{H}), 2.04-2.18(\mathrm{~m}, 1 \mathrm{H}), 1.85-1.95$ (m, 2H), 1.81 (br. s., 1H), 1.66-1.76 (m, 1H), 1.62 (d, $J=3.51 \mathrm{~Hz}, 6 \mathrm{H}), 1.44-1.59$ (m, 2H), 1.28 $1.38(\mathrm{~m}, 5 \mathrm{H}), 0.87-0.95(\mathrm{~m}, 6 \mathrm{H}), 0.85(\mathrm{~d}, J=6.24 \mathrm{~Hz}, 3 \mathrm{H}), 0.79-0.83(\mathrm{~m}, 3 \mathrm{H}), 0.77$ (d, $J=6.63 \mathrm{~Hz}$, $3 \mathrm{H})$. HRMS (m/z): calculated for $\mathrm{C}_{39} \mathrm{H}_{56} \mathrm{~N}_{6} \mathrm{NaO}_{5}[\mathrm{M}+\mathrm{Na}]^{+} 711.4204$; found 711.4216.

\section{tert-butyl3-((4-((4S,7R,10S)-4-benzyl-10-isobutyl-7-isopropyl-13- methyl-3,6,9,12-tetraoxo-1-phenyl-2-oxa-5,8,11-triazatetradecan-13-yl)- 1H-1,2,3-triazol-1-yl)methyl)pyrrolidine-1-carboxylate (6z)}

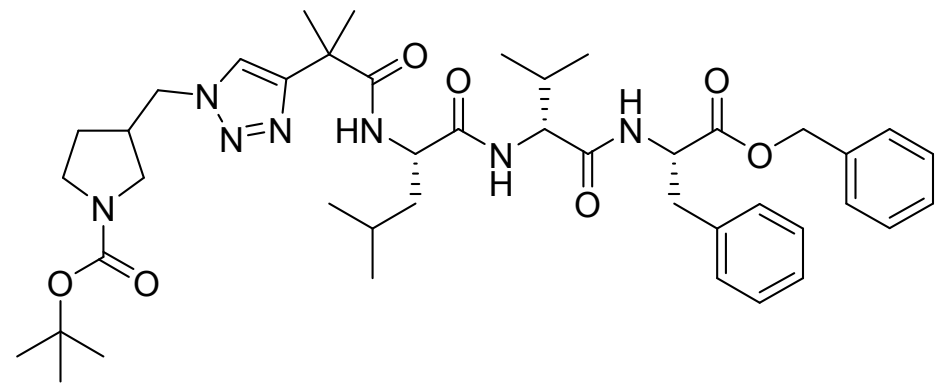

Following GP2, the title compound was prepared as a colorless oil (18 mg, 46\%) starting from tert-butyl 3-(((methylsulfonyl)oxy)methyl)pyrrolidine-1-carboxylate and alkyne (1). Note: for purification, used $\mathrm{KMnO}_{4}$ stain for visualization of product. ${ }^{1} \mathrm{H}$ NMR $(400 \mathrm{MHz}$, CHLOROFORM-d) $\delta 7.41$ (s, 1H), 7.32-7.38 (m, 3H), 7.28 (d, $J=3.12 \mathrm{~Hz}, 1 \mathrm{H}), 7.26$ (br. s., 1H), 7.18-7.25 (m, 3H), 7.10 (s, 1H), 7.03 (dd, $J=2.34,7.02 \mathrm{~Hz}, 2 \mathrm{H}), 6.65-6.73(\mathrm{~m}, 1 \mathrm{H}), 6.34-6.48$ (m, $1 \mathrm{H}), 5.12$ (q, $J=12.10 \mathrm{~Hz}, 2 \mathrm{H}), 4.87(\mathrm{~d}, J=7.80 \mathrm{~Hz}, 1 \mathrm{H}), 4.34$ (t, $J=8.20 \mathrm{~Hz}, 3 \mathrm{H}), 4.17(\mathrm{~s}, 1 \mathrm{H})$, 3.41-3.62 (m, 2H), 3.27-3.40 (m, 1H), 3.10 (d, $J=6.24 \mathrm{~Hz}, 3 \mathrm{H}), 2.70-2.83(\mathrm{~m}, 1 \mathrm{H}), 2.06-2.15$ (m, $1 \mathrm{H}), 1.94-2.04(\mathrm{~m}, 1 \mathrm{H}), 1.65-1.81(\mathrm{~m}, 3 \mathrm{H}), 1.50-1.65(\mathrm{~m}, 8 \mathrm{H}), 1.46(\mathrm{~s}, 9 \mathrm{H}), 0.90$ (d, $J=6.63 \mathrm{~Hz}$, $3 \mathrm{H}), 0.86(\mathrm{~d}, J=6.63 \mathrm{~Hz}, 3 \mathrm{H}), 0.82(\mathrm{~d}, J=7.02 \mathrm{~Hz}, 3 \mathrm{H}), 0.78(\mathrm{dd}, J=1.56,7.02 \mathrm{~Hz}, 3 \mathrm{H})$. HRMS $(\mathrm{m} / \mathrm{z})$ : calculated for $\mathrm{C}_{43} \mathrm{H}_{62} \mathrm{~N}_{7} \mathrm{O}_{7}[\mathrm{M}+\mathrm{H}]^{+}$788.4705; found 788.4709.

\section{Click library examples (Table 1.):}

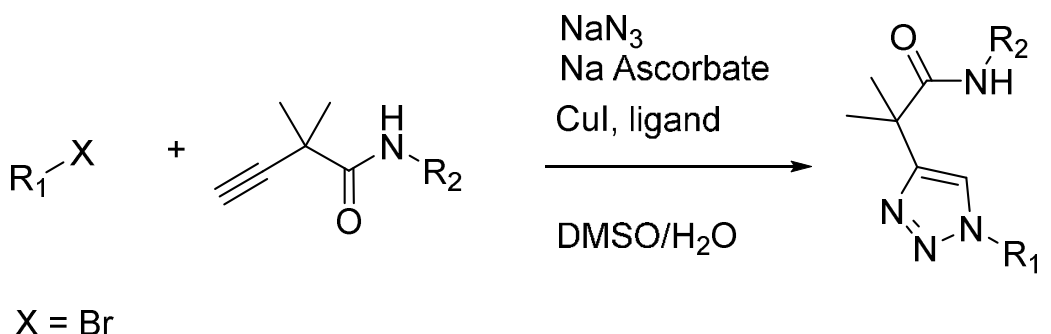

\section{General library procedure:}

The following stock solutions were prepared: 
DMSO solution for each of 4 alkynes - alkyne $(0.3 \mathrm{mmol})$, N1,N2-dimethylcyclohexane-1,2diamine (6.4 mg, $0.03 \mathrm{mmol}$ ) into $1.2 \mathrm{ml}$ DMSO.

Aqueous - sodium ascorbate $(6 \mathrm{mg}, 0.03 \mathrm{mmol})$, sodium azide $(20.8 \mathrm{mg}, 0.319 \mathrm{mmol})$ dissolved into $0.27 \mathrm{ml}$ water.

$\mathrm{CuI}(5.8 \mathrm{mg}, 0.03 \mathrm{mmol})$ in $0.09 \mathrm{ml} \mathrm{DMSO}$

To each vial containing the halide ( $50 \mathrm{umol}, 1 \mathrm{eq}$ ) was added $200 \mathrm{ul}$ of an alkyne stock solution, $45 \mathrm{ul}$ of the aqueous stock solution and $15 \mathrm{ul}$ of the $\mathrm{CuI}$ stock solution giving a 5:1 DMSO/water mixture. The reactions were de-gassed and filled with nitrogen $3 \mathrm{x}^{\prime} \mathrm{s}$ and stirred at $70 \mathrm{C}(5.5 \mathrm{~h})$ then stirred at ambient temperature for $14 \mathrm{~h}$. At which time the reaction was cooled to RT. Added $1 \mathrm{ml}$ water cooled in ice bath, extracted with $3 \mathrm{ml}$ ethyl acetate and concentrated. The crude oils were dissolved into $1 \mathrm{ml}$ DMSO and purified by HPLC (mass triggered), Atlantis dC18 $5 \mu \mathrm{m}, 4.6 \times 50 \mathrm{~mm}$ column, acetonitrile/water $/ 0.05 \%$ TFA, 5 minute run time, $2 \mathrm{ml} / \mathrm{min}$, starting gradient was 95/5 (ACN/water).

\section{Methyl(2-(1-(6-methoxypyridin-3-yl)-1H-1,2,3-triazol-4-yl)-2- methylpropanoyl)-L-leucyl-L-valyl-L-tyrosinate (6c)}<smiles>COC(=O)[C@H](Cc1ccc(O)cc1)NC(=O)[C@H](NC(=O)[C@H](CC(C)C)NC(=O)C(C)(C)c1cn(-c2ccc(OC)nc2)nn1)C(C)C</smiles>

(66\%) HRMS (m/z): calculated for $\mathrm{C}_{33} \mathrm{H}_{46} \mathrm{~N}_{7} \mathrm{O}_{7}[\mathrm{M}+\mathrm{H}]^{+}$652.3453; found 652.3462 .

\section{Benzyl(2-(1-(6-methoxypyridin-3-yl)-1H-1,2,3-triazol-4-yl)-2- methylpropanoyl)-L-phenylalanyl-L-alanyl-L-prolinate (6d)}

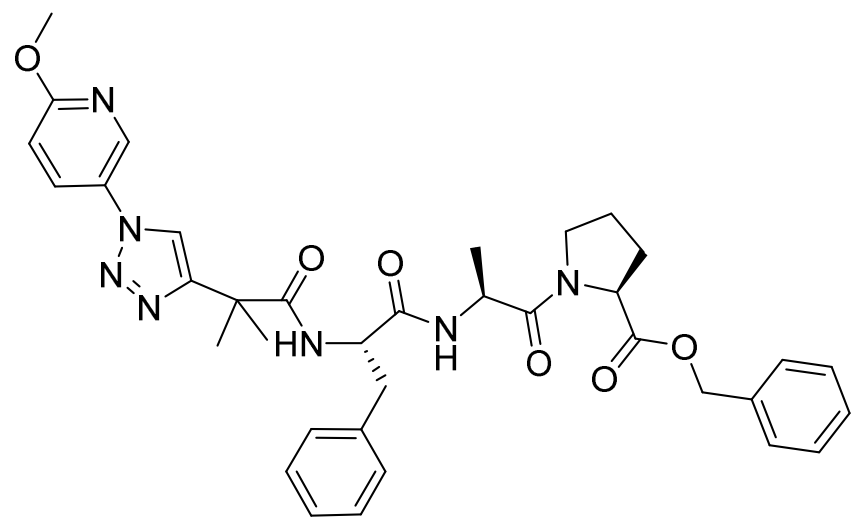

(63\%) HRMS (m/z): calculated for $\mathrm{C}_{36} \mathrm{H}_{42} \mathrm{~N}_{7} \mathrm{O}_{6}[\mathrm{M}+\mathrm{H}]^{+}$668.3191; found 668.3194 .

\section{Benzyl(2-methyl-2-(1-(quinolin-6-yl)-1H-1,2,3-triazol-4-yl)propanoyl)-}




\section{L-leucyl-L-valyl-L-phenylalaninate (6e)}<smiles>CC(C)C[C@H](NC(=O)C(C)(C)c1cn(-c2ccc3ncccc3c2)nn1)C(=O)N[C@@H](C(=O)N[C@@H](Cc1ccccc1)C(=O)OCc1ccccc1)C(C)C</smiles>

(56\%) HRMS (m/z): calculated for $\mathrm{C}_{42} \mathrm{H}_{49} \mathrm{~N}_{7} \mathrm{NaO}_{5}[\mathrm{M}+\mathrm{Na}]^{+}$754.3687; found 754.3689.

Methyl(2-methyl-2-(1-(quinolin-6-yl)-1H-1,2,3-triazol-4-yl)propanoyl)L-leucyl-L-valyl-L-tyrosinate (6f)<smiles>COC(=O)[C@H](Cc1ccc(O)cc1)NC(=O)[C@H](NC(=O)[C@H](CC(C)C)NC(=O)C(C)(C)c1cn(-c2ccc3ncccc3c2)nn1)C(C)C</smiles>

(80\%) HRMS (m/z): calculated for $\mathrm{C}_{36} \mathrm{H}_{46} \mathrm{~N}_{7} \mathrm{O}_{6}[\mathrm{M}+\mathrm{H}]^{+}$672.3504; found 672.3518 .

\section{Benzyl(2-methyl-2-(1-(quinolin-6-yl)-1H-1,2,3-triazol-4-yl)propanoyl)-} L-phenylalanyl-L-alanyl-L-prolinate (6g)

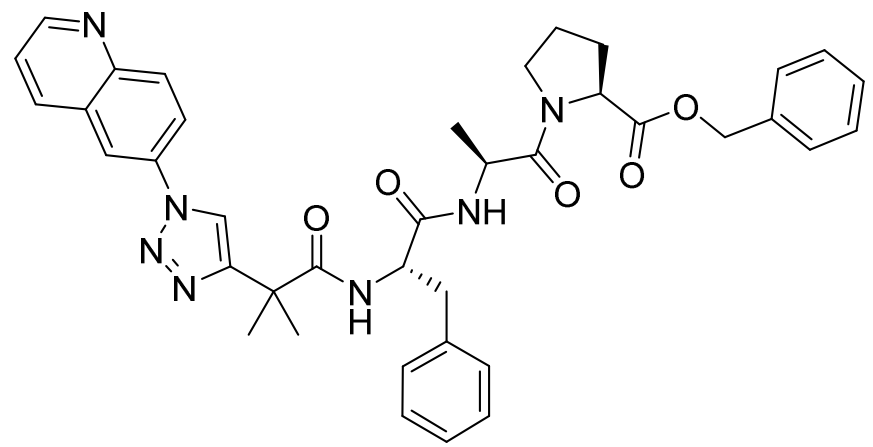

(73\%) HRMS (m/z): calculated for $\mathrm{C}_{39} \mathrm{H}_{42} \mathrm{~N}_{7} \mathrm{O}_{5}[\mathrm{M}+\mathrm{H}]^{+}$688.3242; found 688.3239. 
Benzyl (2-methyl-2-(1-(quinolin-6-yl)-1H-1,2,3-triazol-4-yl)propanoyl)D-alanyl-L-alanyl-L-prolinate (6h)<smiles>CC(NC(=O)C(C)(C)c1cn(-c2ccc3ncccc3c2)nn1)C(=O)NC(C)C(=O)N1CCCC1C(=O)OCc1ccccc1</smiles>

(80\%) HRMS (m/z): calculated for $\mathrm{C}_{33} \mathrm{H}_{38} \mathrm{~N}_{7} \mathrm{O}_{5}[\mathrm{M}+\mathrm{H}]^{+}$612.2929; found 612.2932.

Methyl(2-(1-(1H-benzo[d]imidazol-5-yl)-1H-1,2,3-triazol-4-yl)-2methylpropanoyl)-L-leucyl-L-valyl-L-tyrosinate (6k)<smiles>COC(=O)[C@H](Cc1ccc(O)cc1)NC(=O)[C@H](NC(=O)[C@H](CC(C)C)NC(=O)C(C)(C)c1cn(-c2ccc3[nH]cnc3c2)nn1)C(C)C</smiles>

(43\%) HRMS (m/z): calculated for $\mathrm{C}_{34} \mathrm{H}_{45} \mathrm{~N}_{8} \mathrm{O}_{6}[\mathrm{M}+\mathrm{H}]^{+}$661.3457; found 661.3461 .

Benzyl(2-(1-(1H-benzo[d]imidazol-5-yl)-1H-1,2,3-triazol-4-yl)-2methylpropanoyl)-L-phenylalanyl-L-alanyl-L-prolinate (6I)<smiles>CC(NC(=O)[C@H](Cc1ccccc1)NC(=O)C(C)(C)c1cn(-c2ccc3[nH]cnc3c2)nn1)C(=O)N1CCC[C@H]1C(=O)OCc1ccccc1</smiles>

(46\%) HRMS (m/z): calculated for $\mathrm{C}_{37} \mathrm{H}_{41} \mathrm{~N}_{8} \mathrm{O}_{5}[\mathrm{M}+\mathrm{H}]^{+}$677.3194; found 677.3204 . 
Benzyl(2-methyl-2-(1-(2-methylpyridin-4-yl)-1H-1,2,3-triazol-4yl)propanoyl)-L-leucyl-D-valyl-L-phenylalaninate (6m)<smiles>Cc1cc(-n2cc(C(C)(C)C(=O)N[C@@H](CC(C)C)C(=O)NC(C(=O)N[C@H](Cc3ccccc3)C(=O)OCc3ccccc3)C(C)C)nn2)ccn1</smiles>

(36\%) HRMS (m/z): calculated for $\mathrm{C}_{39} \mathrm{H}_{50} \mathrm{~N}_{7} \mathrm{O}_{5}[\mathrm{M}+\mathrm{H}]^{+}$696.3868; found 696.3871 .

Methyl(2-methyl-2-(1-(2-methylpyridin-4-yl)-1H-1,2,3-triazol-4yl)propanoyl)-L-leucyl-L-valyl-L-tyrosinate (6n)<smiles>COC(=O)[C@H](Cc1ccc(O)cc1)NC(=O)[C@H](NC(=O)[C@H](CC(C)C)NC(=O)C(C)(C)c1cn(-c2ccnc(C)c2)nn1)C(C)C</smiles>

(32\%) HRMS (m/z): calculated for $\mathrm{C}_{33} \mathrm{H}_{46} \mathrm{~N}_{7} \mathrm{O}_{6}[\mathrm{M}+\mathrm{H}]^{+}$636.3504; found 636.3502.

Benzyl(2-methyl-2-(1-(2-methylpyridin-4-yl)-1H-1,2,3-triazol-4yl)propanoyl)-L-phenylalanyl-L-alanyl-L-prolinate (6o)

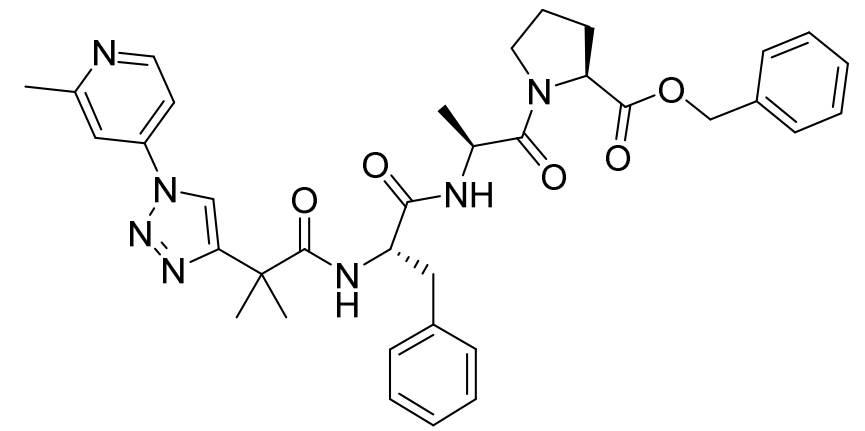

(34\%) HRMS (m/z): calculated for $\mathrm{C}_{36} \mathrm{H}_{42} \mathrm{~N}_{47} \mathrm{O}_{5}[\mathrm{M}+\mathrm{H}]^{+}$652.3242; found 652.3239. 
Benzyl(2-(1-benzyl-1H-1,2,3-triazol-4-yl)-2-methylpropanoyl)-L-leucylL-valyl-L-phenylalaninate (6p)<smiles>CC(C)C[C@H](NC(=O)C(C)(C)c1cn(Cc2ccccc2)nn1)C(=O)N[C@@H](C(=O)N[C@@H](Cc1ccccc1)C(=O)OCc1ccccc1)C(C)C</smiles>

(67\%) HRMS (m/z): calculated for $\mathrm{C}_{40} \mathrm{H}_{51} \mathrm{~N}_{6} \mathrm{O}_{5}[\mathrm{M}+\mathrm{H}]^{+}$695.3915; found 695.3934.

Methyl(2-(1-benzyl-1H-1,2,3-triazol-4-yl)-2-methylpropanoyl)-L-leucylL-valyl-L-tyrosinate (6q)<smiles>COC(=O)[C@H](Cc1ccc(O)cc1)NC(=O)[C@@H](NC(=O)[C@H](CC(C)C)NC(=O)C(C)(C)c1cn(Cc2ccccc2)nn1)C(C)C</smiles>

(62\%) HRMS (m/z): calculated for $\mathrm{C}_{34} \mathrm{H}_{47} \mathrm{~N}_{6} \mathrm{O}_{6}[\mathrm{M}+\mathrm{H}]^{+}$635.3552; found 635.3568.

Benzyl(2-(1-benzyl-1H-1,2,3-triazol-4-yl)-2-methylpropanoyl)-Lphenylalanyl-L-alanyl-L-prolinate (6r)

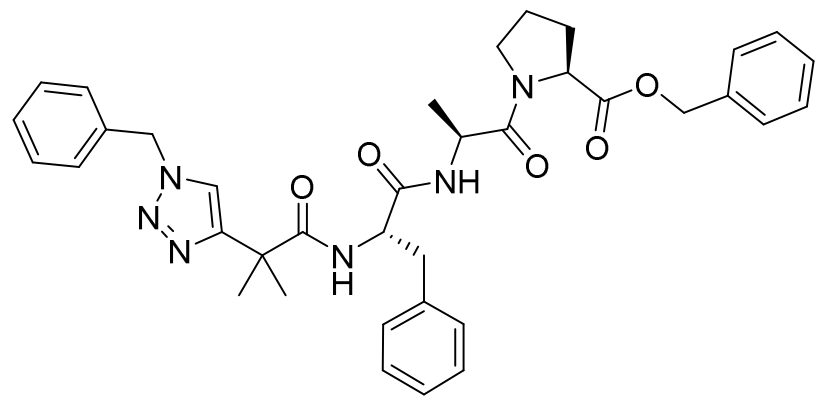

(57\%) HRMS (m/z): calculated for $\mathrm{C}_{37} \mathrm{H}_{43} \mathrm{~N}_{6} \mathrm{O}_{5}[\mathrm{M}+\mathrm{H}]^{+}$651.3289; found 651.3295. 
Benzyl(2-(1-benzyl-1H-1,2,3-triazol-4-yl)-2-methylpropyl)-L-alanyl-Lalanyl-L-prolinate $(6 s)$<smiles>CC(NC(C)(C)c1cn(Cc2ccccc2)nn1)C(=O)NC(C)C(=O)N1CCCC1C(=O)OCc1ccccc1</smiles>

(60\%) HRMS (m/z): calculated for $\mathrm{C}_{31} \mathrm{H}_{38} \mathrm{~N}_{6} \mathrm{NaO}_{65}[\mathrm{M}+\mathrm{Na}]^{+}$597.2796; found 597.2806.

Methyl(2-methyl-2-(1-phenethyl-1H-1,2,3-triazol-4-yl)propanoyl)-Lleucyl-L-valyl-L-tyrosinate (6u)<smiles>COC(=O)[C@H](Cc1ccc(O)cc1)NC(=O)[C@H](NC(=O)[C@H](CC(C)C)NC(=O)C(C)(C)c1cn(CCc2ccccc2)nn1)C(C)C</smiles>

(68\%) HRMS (m/z): calculated for $\mathrm{C}_{20} \mathrm{H}_{26} \mathrm{~N}_{4} \mathrm{O}_{4} \mathrm{~S}[\mathrm{M}+\mathrm{H}]^{+} 419.1748$; found 419.1746 .

\section{Benzyl(2-methyl-2-(1-phenethyl-1H-1,2,3-triazol-4-yl)propanoyl)-L-} phenylalanyl-L-alanyl-L-prolinate (6v)

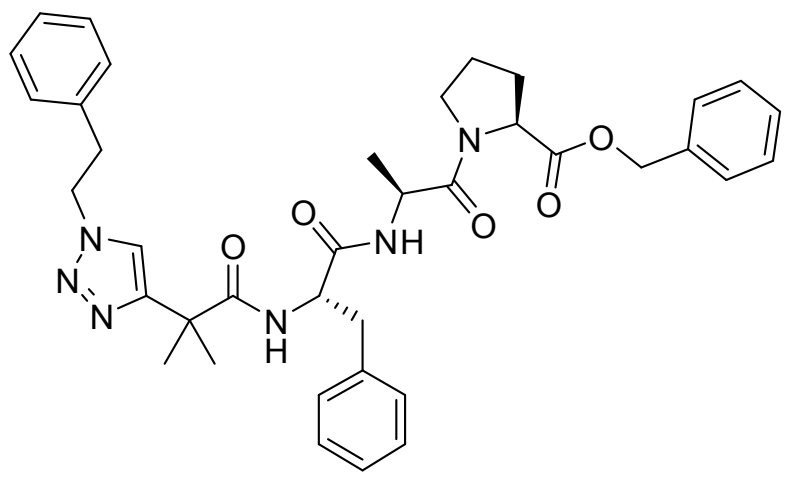

(74\%) HRMS (m/z): calculated for $\mathrm{C}_{37} \mathrm{H}_{41} \mathrm{~N}_{8} \mathrm{O}_{5}[\mathrm{M}+\mathrm{H}]^{+}$665.3459; found 665.3446. 


\section{Benzyl(2-methyl-2-(1-phenethyl-1H-1,2,3-triazol-4-yl)propanoyl)-L- alanyl-L-alanyl-L-prolinate (6w)}

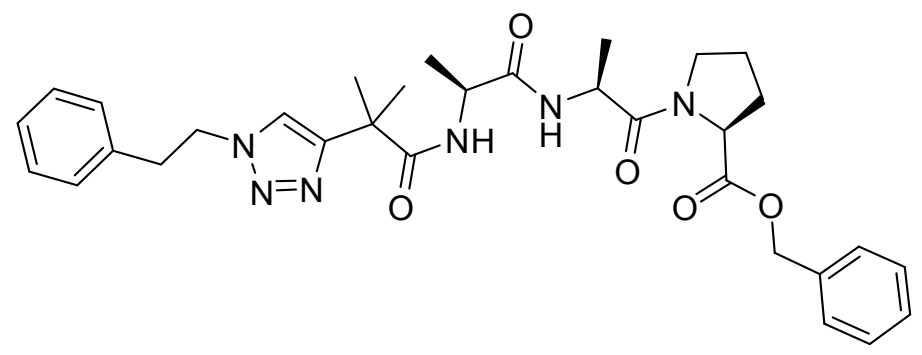

(72\%) HRMS (m/z): calculated for $\mathrm{C}_{32} \mathrm{H}_{41} \mathrm{~N}_{6} \mathrm{O}_{5}[\mathrm{M}+\mathrm{H}]^{+}$589.3133; found 589.3158.

\section{NMR Spectra:}
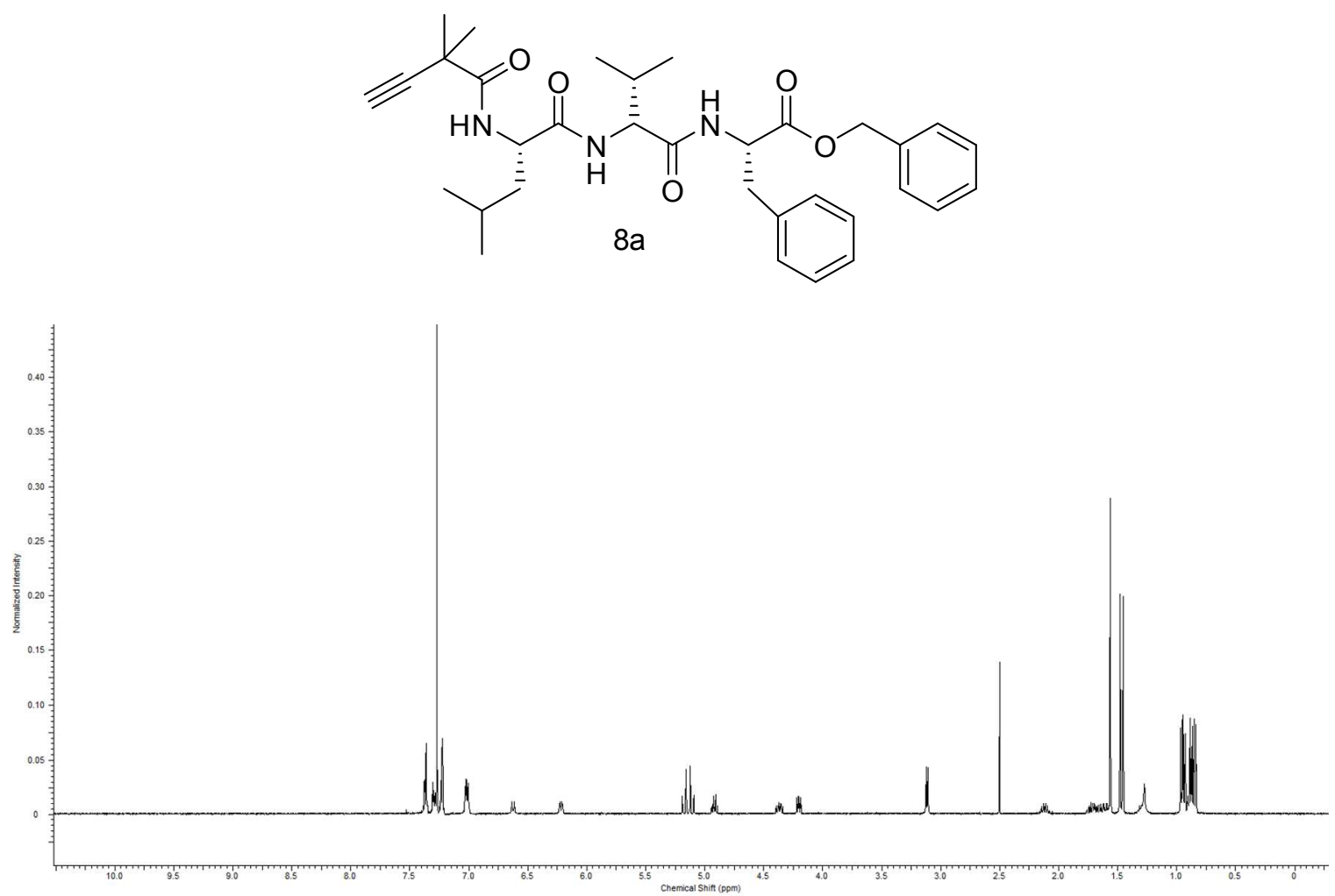

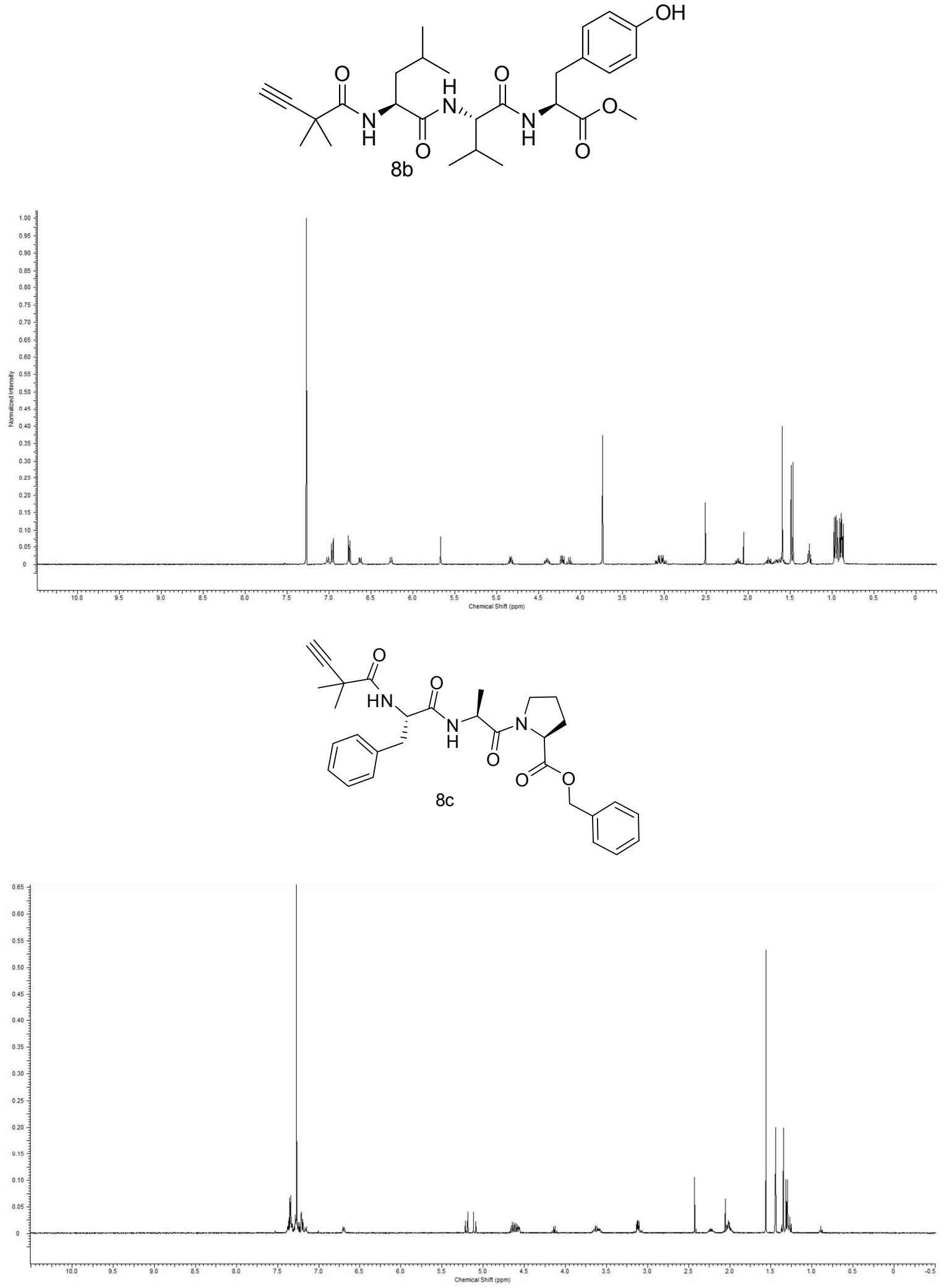

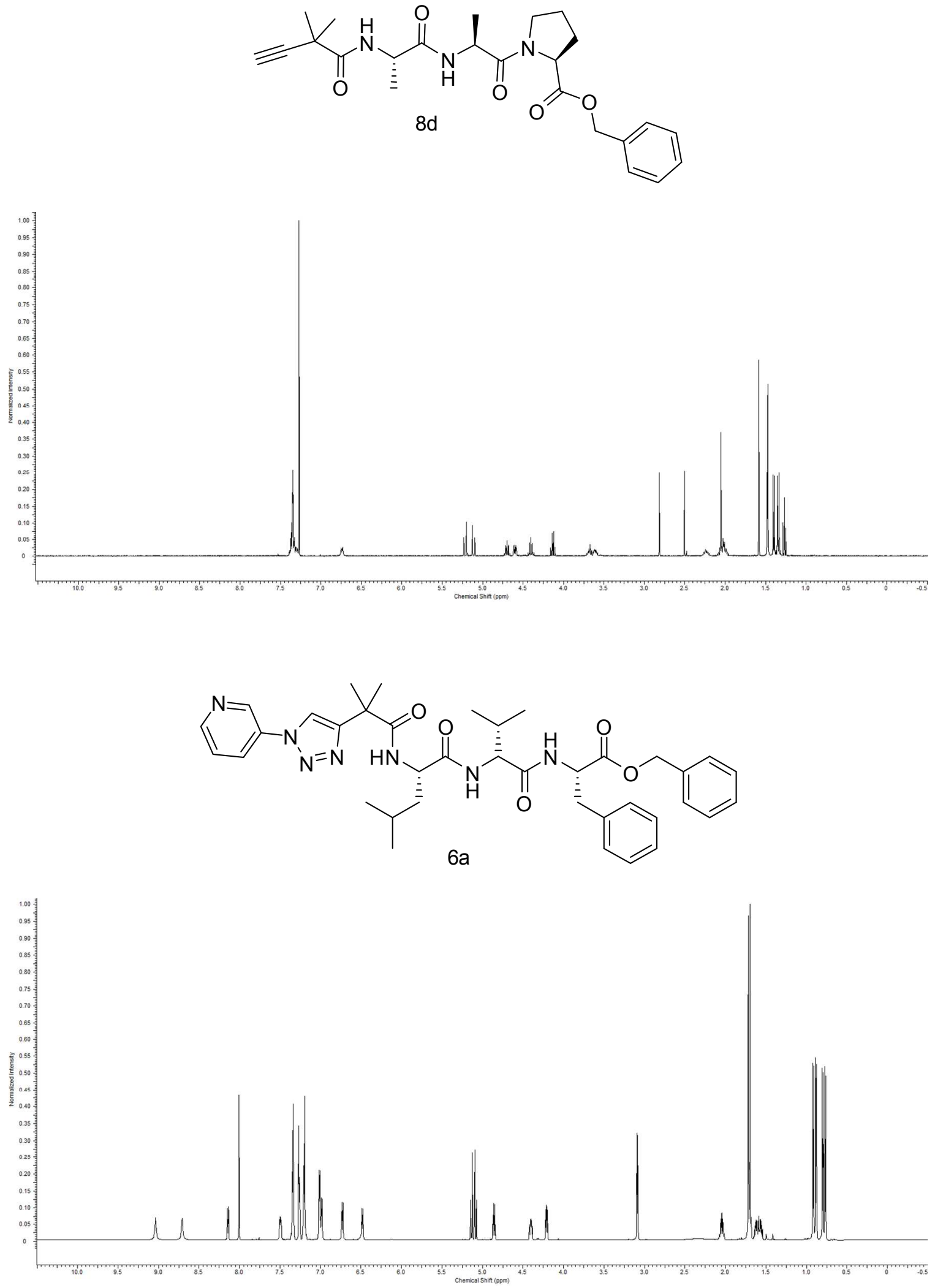
Supporting Information

S22
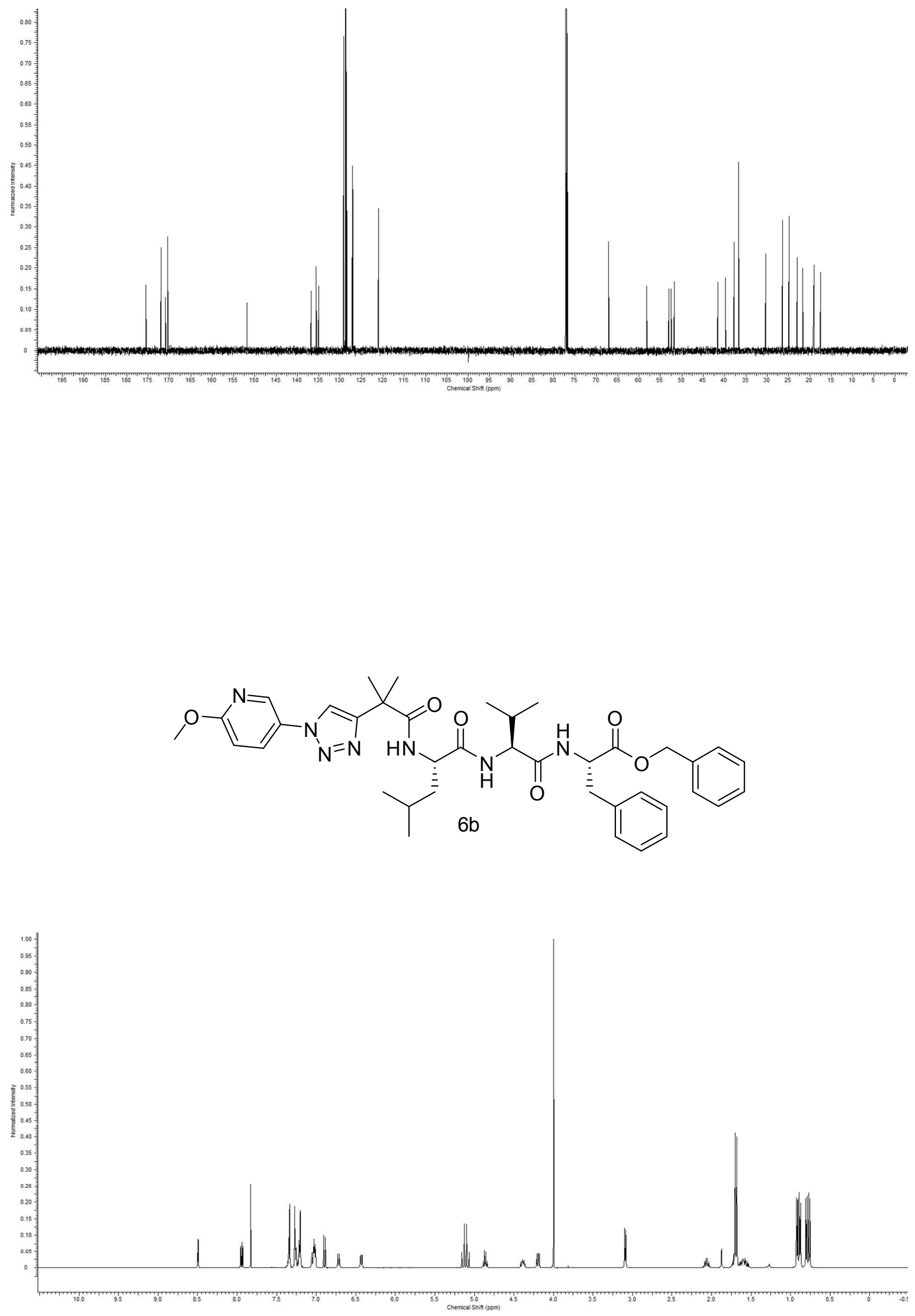

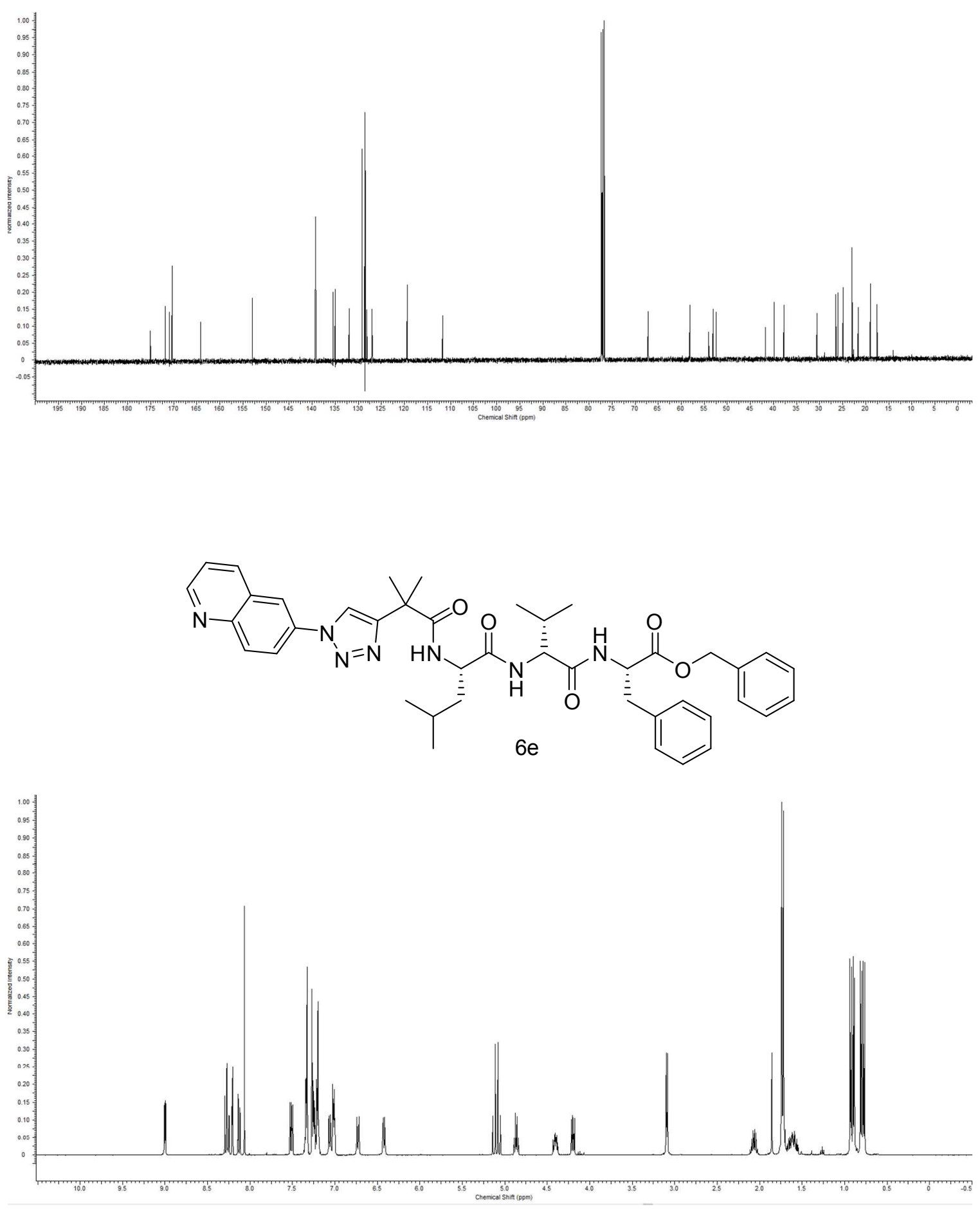

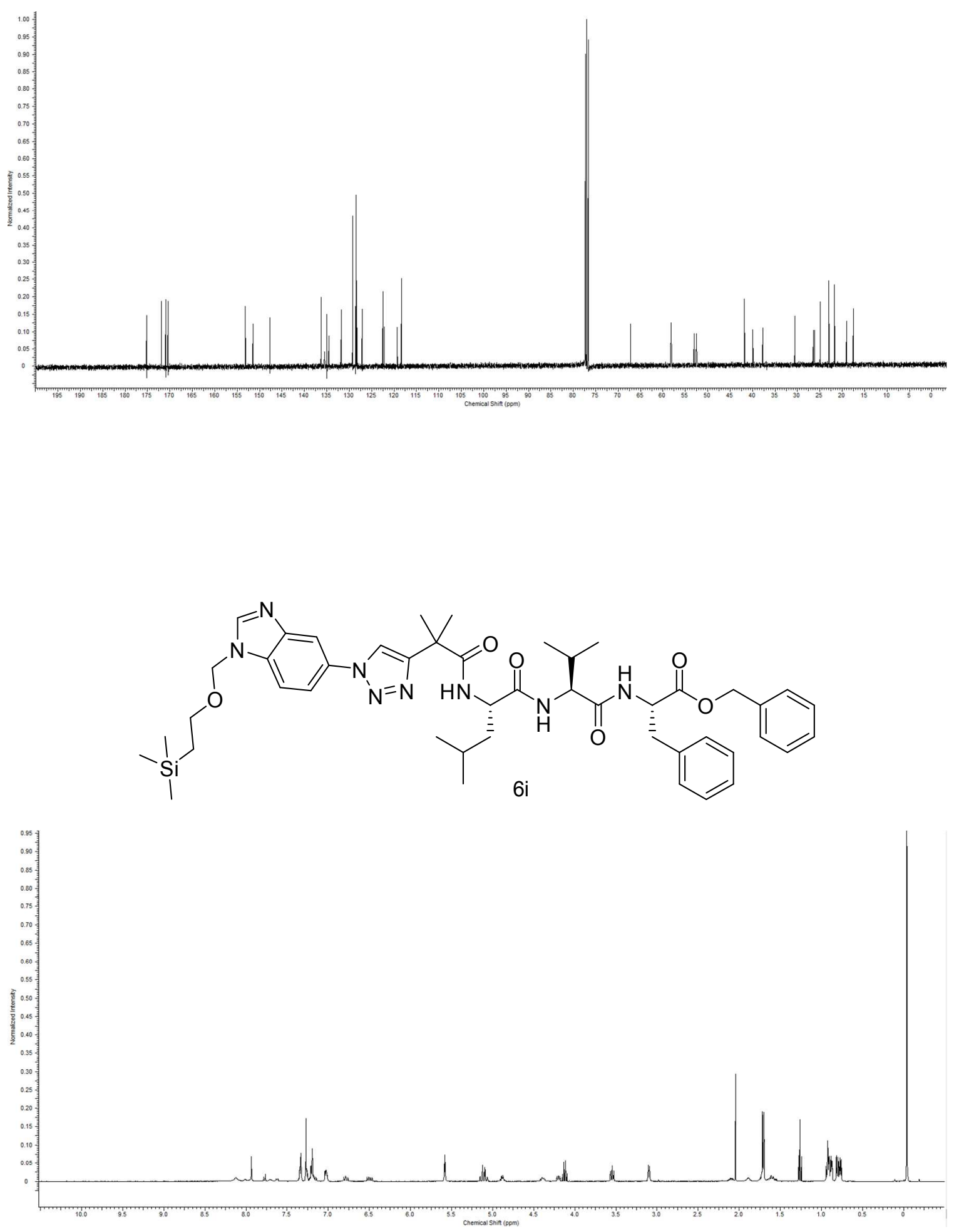

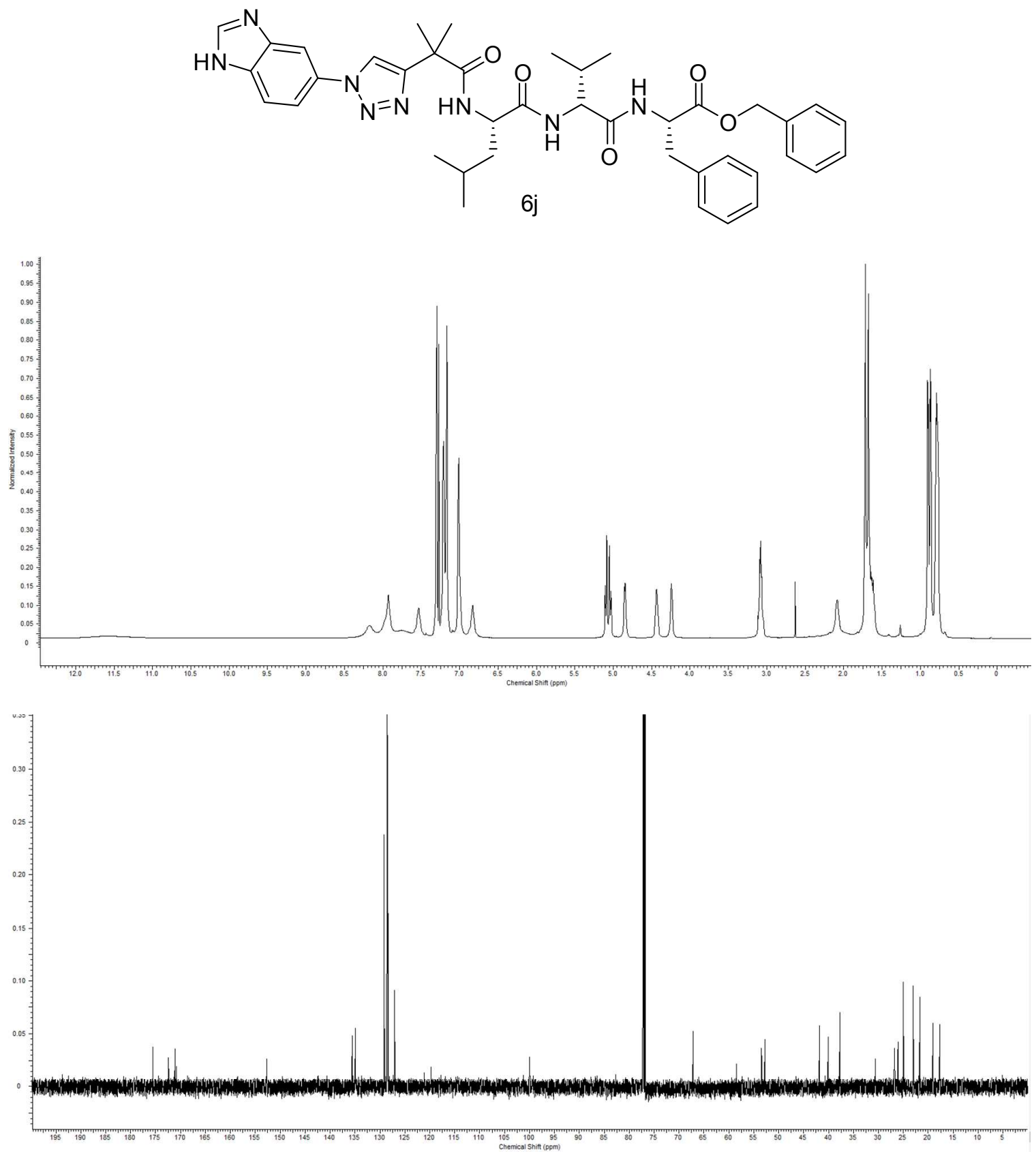

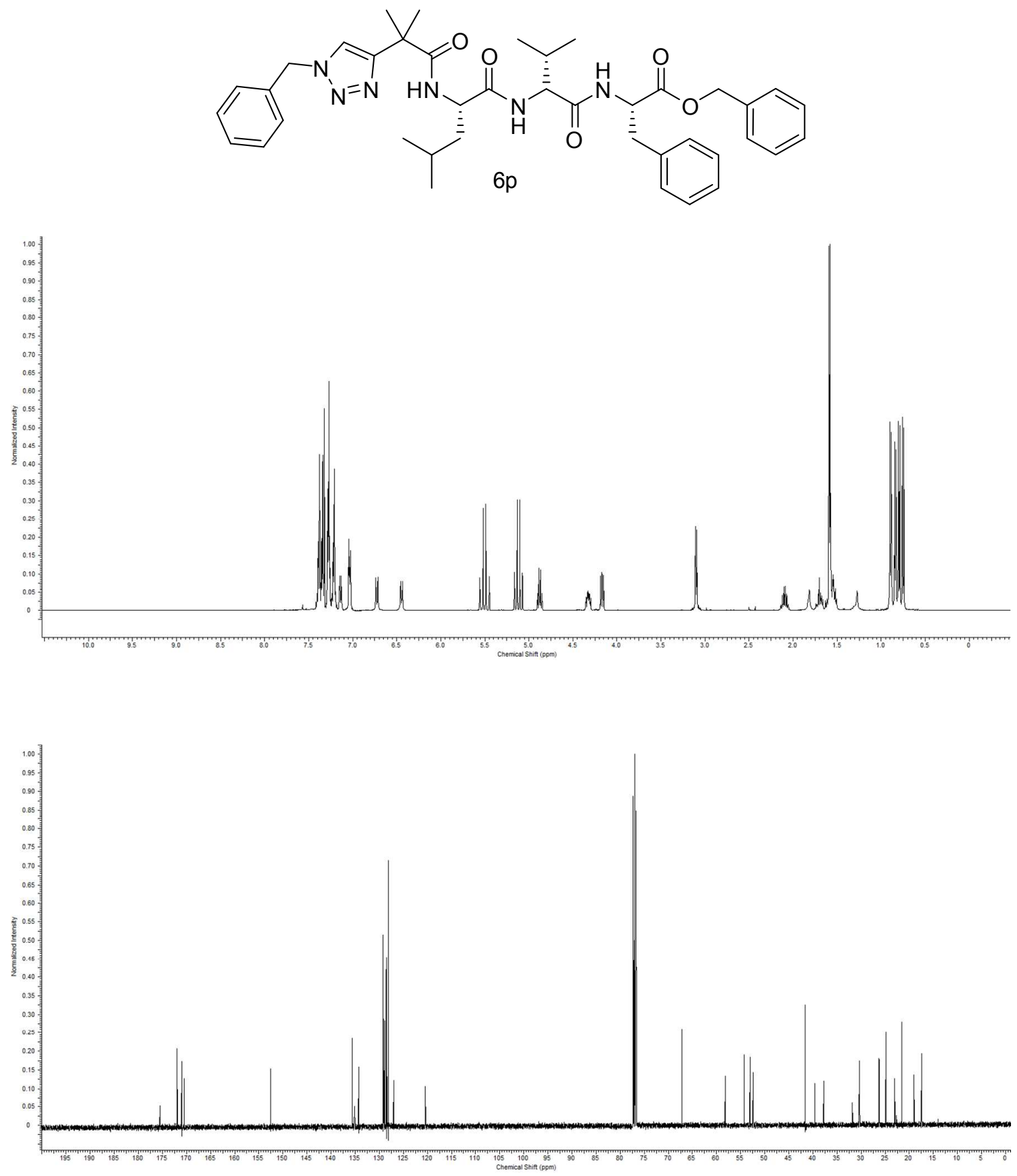

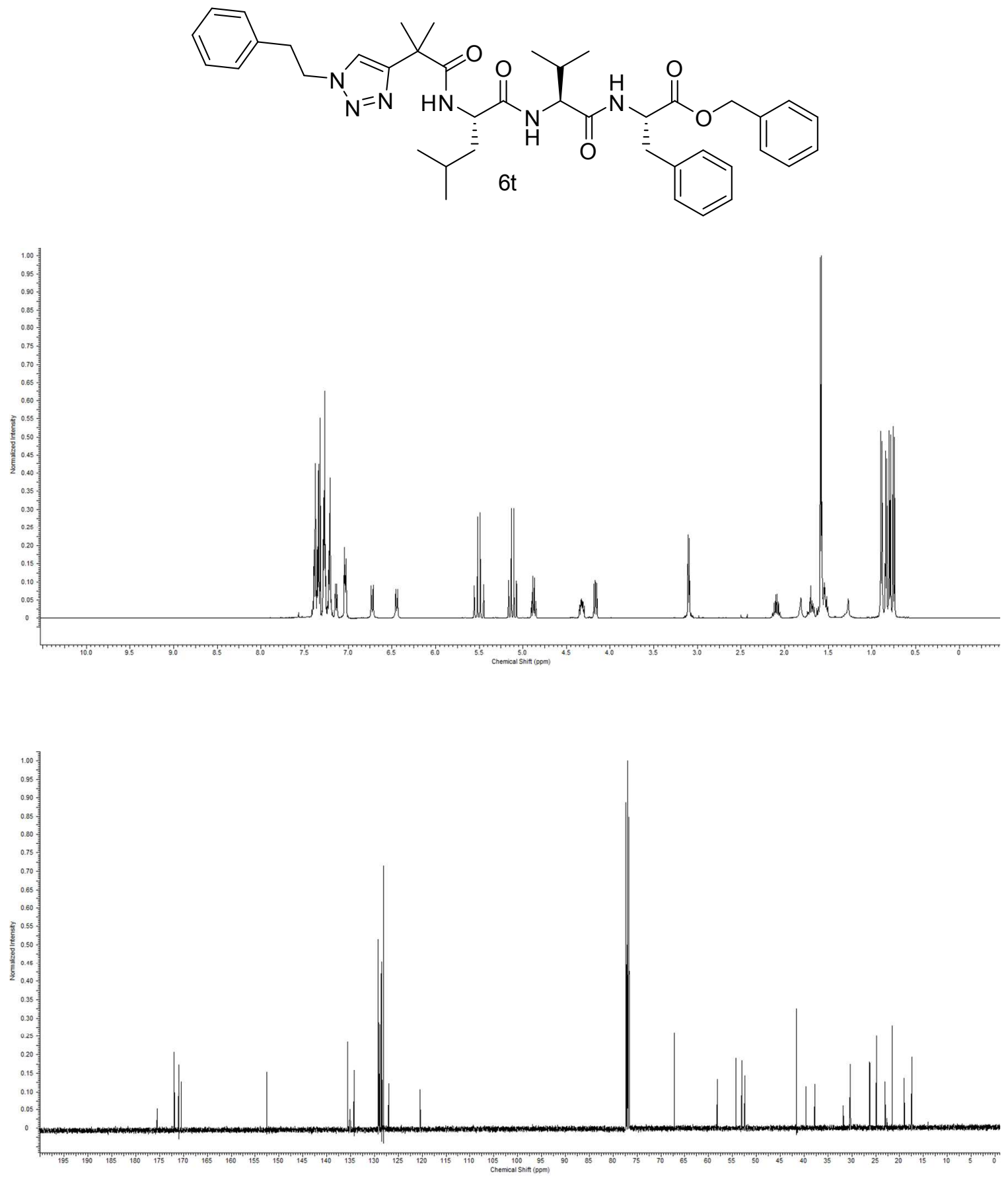

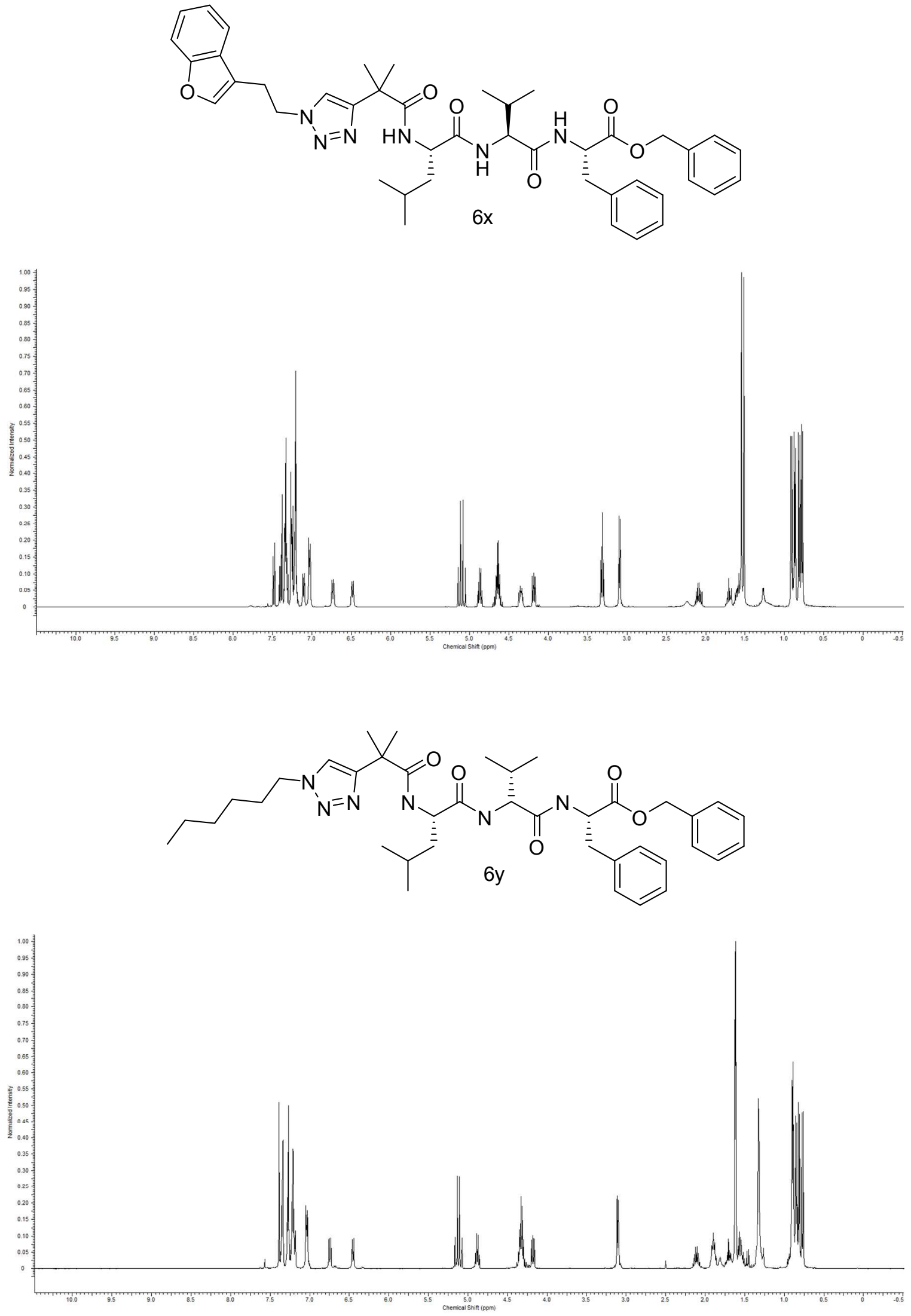

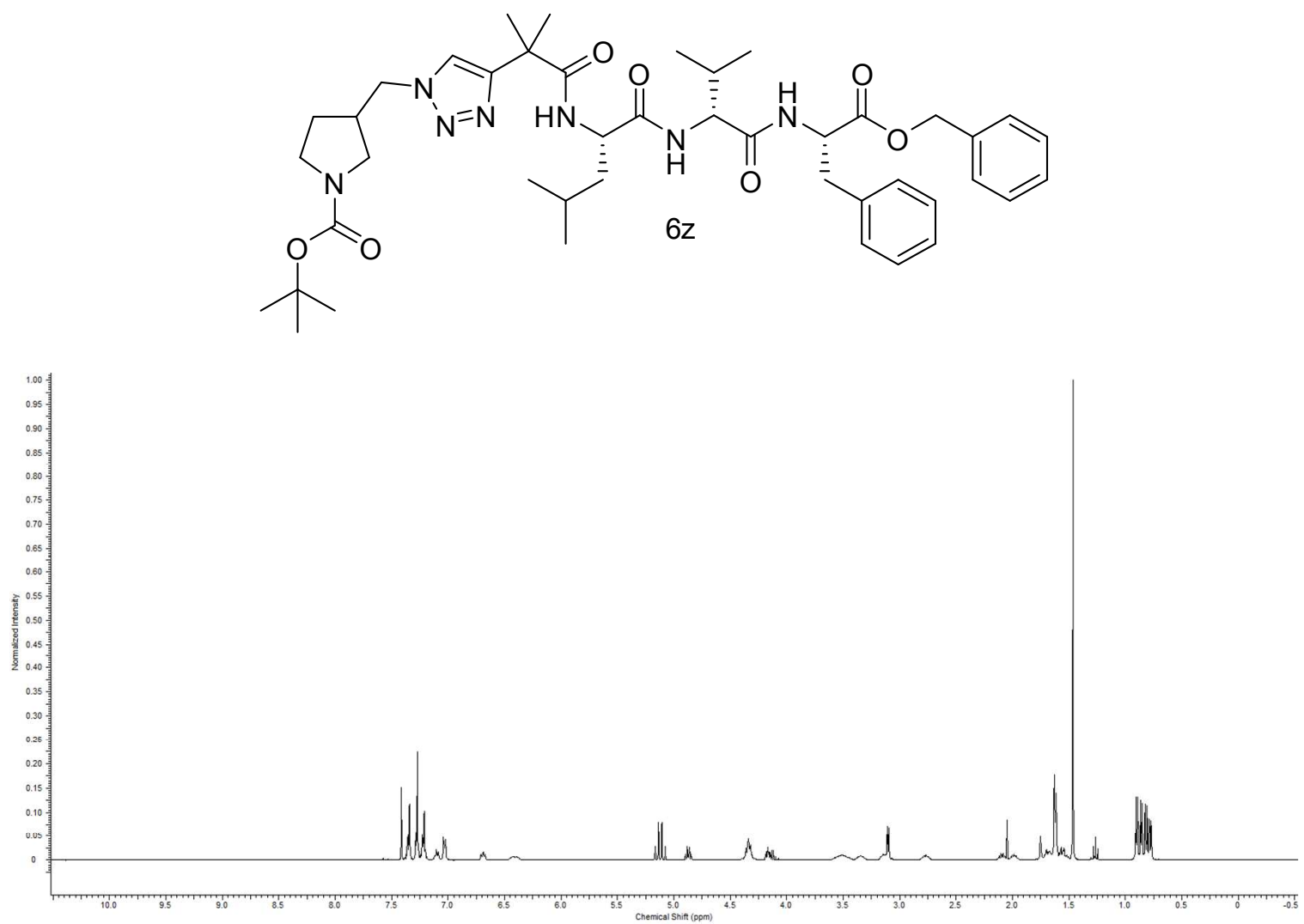